\title{
Analysis of Galactic late-type 0 dwarfs: more constraints on the weak wind problem ${ }^{\star} \star \star$
}

\author{
W. L. F. Marcolino ${ }^{1}$, J.-C. Bouret ${ }^{1}$, F. Martins ${ }^{2}$, D. J. Hillier ${ }^{3}$, T. Lanz ${ }^{4}$, and C. Escolano ${ }^{1}$ \\ 1 LAM-UMR 6110, CNRS \& Univ. de Provence, 38 rue Fréderic Joliot-Curie, 13388 Marseille, France \\ e-mail: wagner.marcolino@oamp.fr \\ 2 GRAAL-UMR 5024, CNRS \& Univ. de Montpellier II, Place Bataillon, 34095 Montpellier, France \\ 3 Department of Physics and Astronomy, University of Pittsburgh, Pittsburgh, PA 15260, USA \\ 4 Department of Astronomy, University of Maryland, College Park, MD 20742, USA
}

Received 4 November 2008 / Accepted 17 February 2009

\section{ABSTRACT}

\begin{abstract}
Aims. We investigate the stellar and wind properties of a sample of late-type $\mathrm{O}$ dwarfs. Previous analyses of such stars have found very low mass-loss rates; rates much lower than predicted by theory (the weak wind problem).

Methods. Far-UV to optical spectra of five Galactic O stars were analyzed: HD 216898 (O9IV/O8.5V), HD 326329 (O9V), HD 66788 $(\mathrm{O} 8 \mathrm{~V} / \mathrm{O} 9 \mathrm{~V}), \zeta \mathrm{Oph}(\mathrm{O} 9.5 \mathrm{Vnn})$, and HD $216532(\mathrm{O} 8.5 \mathrm{~V}((\mathrm{n})))$. We used a grid of TLUSTY models to obtain effective temperatures, gravities, rotational velocities, and to identify wind lines. Wind parameters for each object were obtained using expanding atmosphere models calculated with the CMFGEN code.

Results. The spectra of our sample have primarily a photospheric origin. A weak wind signature is seen in C IV $\lambda \lambda 1548$, 1551, from which mass-loss rates consistent with previous CMFGEN results for O8-O9V stars were derived $\left(\sim 10^{-10}-10^{-9} M_{\odot} \mathrm{yr}^{-1}\right)$. A discrepancy of roughly two orders of magnitude is found between these mass-loss rates and the values predicted by theory $\left(\dot{M}_{\text {Vink }}\right)$, confirming a breakdown or a steepening of the modified wind momentum-luminosity relation at $\log L_{\star} / L_{\odot} \lesssim 5.2$. We have estimated the carbon abundance for the stars of our sample and concluded that its value cannot be reduced to sufficiently small values to solve the weak wind problem. Upper limits on $\dot{M}$ were established for all objects using lines of different ions: P v $\lambda \lambda 1118,1128, \mathrm{C}$ III $\lambda 1176$, N v $\lambda \lambda 1239,1243$, Si IV $\lambda \lambda 1394,1403$, and N IV $\lambda 1718$. All the values obtained are in disagreement with theoretical predictions, bringing support to the reality of weak winds. Together with C IV $\lambda \lambda 1548,1551$, the use of $\mathrm{N} v \lambda \lambda 1239,1243$ results in the lowest mass-loss rates: the upper limits indicate that $\dot{M}$ must be less than about -1.0 dex $\dot{M}_{\text {Vink }}$. Upper mass-loss rate limits obtained for other transitions are also low: they indicate that $\dot{M}$ must be less than about $(-0.5 \pm 0.2)$ dex $\dot{M}_{\text {Vink }}$. We studied the behavior of the H $\alpha$ line with different mass-loss rates. For two stars, only models with very low $\dot{M}$ 's provide the best fit to the UV and optical spectra. We also explored ways to fit the observed spectra with the theoretical mass-loss rates. By using large amounts of X-rays, we could reduce the predicted wind emission to the observed levels. However, unrealistic X-ray luminosities had to be used $\left(\log L_{\mathrm{X}} / L_{\mathrm{Bol}} \gtrsim-3.5\right)$. The validity of the models used in our analyses is discussed.
\end{abstract}

Key words. stars: atmospheres - stars: mass-loss - stars: fundamental parameters - stars: early-type

\section{Introduction}

Massive stars of spectral types $\mathrm{O}$ and B play an extremely important role in astrophysics. They possess high effective temperatures $\left(T_{\text {eff }}>10 \mathrm{kK}\right)$, intense ionizing radiation fields and often exhibit strong mass-loss through stellar winds, making their description considerably difficult for atmosphere and stellar evolution models. These stars are known to be progenitors of fascinating objects such as red supergiants (RSGs), luminous blue variables (LBVs), Wolf-Rayet stars (W-Rs), and thus also of some of the most energetic phenomena in the Universe - type II

\footnotetext{
* Based on observations made with the NASA-CNES-CSA Far Ultraviolet Spectroscopic Explorer and by the NASA-ESA-SERC International Ultraviolet Explorer, and retrieved from the Multimission Archive at the Space Telescope Science Institute (MAST). Based on observations collected with the ELODIE spectrograph on the $1.93-\mathrm{m}$ telescope (Observatoire de Haute-Provence, France). Based on observations collected with the FEROS instrument on the ESO $2.2 \mathrm{~m}$ telescope, program 074.D-0300 and 075.D-0061.

$\star \star$ Appendices A and B are only available in electronic form at http://www. aanda.org
}

supernovae and some $\gamma$-ray bursts (Massey 2003; Woosley \& Bloom 2006). They also heavily affect their host galaxies by transferring momentum, energy and enriched chemical elements to the interstellar medium (Abbott 1982; Freyer et al. 2003).

Although they have been studied for decades, the properties, origin and evolution of OB stars still present several observational and theoretical challenges. The dependency of their massloss rates $(\dot{M})$ on the metallicity $(Z)$, for example, as well as their effective temperatures and wind structure (e.g., clumping) have been continually debated in the literature during the last few years (see, e.g., Vink et al. 2001; Martins et al. 2002; Bouret et al. 2005; Puls et al. 2006; Crowther et al. 2006).

Among several interesting issues currently under discussion (for a review see Puls 2008; Hillier 2008) is the so-called weak wind problem, which is associated with late-type $\mathrm{O}$ dwarf stars. Qualitatively, O stars with weak winds present an absorption spectrum, with the exception of a few weak wind lines. In some stars only a weak C IV $\lambda \lambda 1548,1551$ P-Cygni profile is seen. In contrast, mid- and early $\mathrm{O}$ dwarfs can present $\mathrm{P}$-Cygni profiles in lines such as C IV $\lambda \lambda 1548,1551, \mathrm{~N}$ IV $\lambda 1718, \mathrm{~N}$ V $\lambda \lambda 1239,1243$ or O v $\lambda 1371$ (see, e.g., Snow et al. 1994; Escolano et al. 2008). 
Quantitatively, weak wind stars are defined as having massloss rates of less than about $10^{-8} M_{\odot} \mathrm{yr}^{-1}$. Bouret et al. (2003) were one of the first to suggest such low values after analyzing O dwarfs in the HII region NGC 346 in the SMC. The spectra of three objects of their sample could only be reproduced by models using mass-loss rates of $\sim 10^{-10}$ to $10^{-9} M_{\odot} \mathrm{yr}^{-1}$. Similar results were found by Martins et al. (2004) for four O dwarfs in the compact star formation region N81, also in the SMC. Later, weak winds were also found in some Galactic $\mathrm{O}$ dwarfs, demonstrating that they are not the result of an environmental (i.e., metallicity) effect (Martins et al. 2005a). Despite these findings very low $\dot{M}$ values are not of general consensus.

There are many interesting questions that $\mathrm{O}$ stars with very low mass-loss rates pose. Mass-loss is a fundamental ingredient in stellar evolution models; different values for this parameter alter considerably the way the stars evolve. For example, mass loss changes the rotational structure of a star (decreasing surface and internal velocities) due to the removal of angular momentum combined with internal transport mechanisms (Meynet \& Maeder 2000). Very low $\dot{M}$ 's might thus imply that stars can keep high rotational velocities and get closer to break-up velocities. Low mass-loss rates can also change the way we understand evolution in the LBV and W-R phases. The total mass lost from the main sequence prior to the LBV phase may be much less than currently thought. In order to be consistent with observed masses of hydrogen deficient W-R stars, other intense mass-loss mechanisms must occur (e.g., continuum driven giant eruptions in the LBV phase), or evolutionary time scales (e.g., of the WNL phase) must be changed (see Smith \& Owocki 2006; van Marle et al. 2008), or both. Given these considerations, it is essential that we examine the validity of weak winds.

The low mass-loss rates obtained for late-type $\mathrm{O}$ dwarfs also present challenges for the theory of radiatively driven winds. While state-of-the-art theoretical $\dot{M}$ predictions of Vink et al. $\left(2000,2001 ; \dot{M}_{\text {Vink }}\right)$ present a good match for objects with $\log L / L_{\odot}$ greater than about 5.2 (neglecting clumping), they provide a poor match for late-type, less luminous objects where a discrepancy of up to a factor of one hundred can be found (Martins et al. 2005a). Late O dwarfs data also suggest a breakdown of the modified wind momentum luminosity relation (WLR; Kudritzki \& Puls 2000) or at least a change in its slope (see Fig. 41 of Martins et al. 2005a). All these facts constitute what is now called the weak wind problem ${ }^{1}$.

A possible reason for the aforementioned discrepancies is that mass-loss rate determinations based on UV lines could be incorrect, as was suggested by Mokiem et al. (2007). The use of only one UV diagnostic line (i.e., C IV $\lambda \lambda 1548,1551)$, the abundance of carbon, the effect of X-rays, and the wind ionization structure derived from the models, are claimed to be considerable sources of errors for the derivation of $\dot{M}$. This means that stronger winds could perhaps have been found if appropriate diagnostic tools were used/available. According to Mokiem et al. (2007), three objects which are supposed to be in the weak wind regime ( $\zeta$ Oph, CygOB2\#2, and HD 217086) actually do not show very low mass-loss rates (i.e., $\dot{M}$ 's are consistent with theory) if $\mathrm{H} \alpha$ is used as the diagnostic.

\footnotetext{
${ }^{1}$ Another type of weak wind problem exists, which is the weaker wind signatures of some stars compared to others of the same spectral type (e.g., $\theta^{1}$ Ori C; Walborn \& Panek 1984). Throughout this paper, we consider stars to have "weak winds" when their derived mass-loss rates are significantly lower than the theoretical mass-loss rates predicted by Vink et al. (2000), and are also significantly lower than the mass-loss rates of normal $\mathrm{O}$ stars of earlier spectral types.
}

In order to clarify some of the issues described above, and to get more insight into the weak wind problem, we have analyzed in detail far-UV, UV and optical high-resolution spectra of five Galactic late-type $\mathrm{O}$ dwarf stars. Their stellar and wind physical parameters were obtained using the codes TLUSTY (Hubeny \& Lanz 1995) and CMFGEN (Hillier \& Miller 1998). With this work, the number of O8-9V objects studied by means of state-of-the-art atmosphere models is increased considerably, since only six were previously analyzed (Repolust et al. 2004; Mokiem et al. 2005; Martins et al. 2005a).

The remainder of this paper is organized as follows. In Sect. 2 we describe the way we have selected our targets and the observational material used. A description of the atmosphere codes and the adopted assumptions are given in Sect. 3. The analysis of each object of our sample is presented in Sect. 4. The derived stellar and wind parameters are presented later in Sect. 5 along with comparisons to previous results and theoretical predictions. In Sect. 6 we discuss the carbon abundance and its relation with the mass-loss rate. In Sect. 7 we present estimates for the mass-loss rates of the program stars using other lines besides C IV $\lambda \lambda 1548,1551$. In Sect. 8 we discuss the consequences of our findings and explore a way to have agreement with the observed spectra using the mass-loss rates predicted by theory. Section 9 summarizes the main results found in our study.

\section{Target selection and observations}

The Galactic stars claimed to have weak winds belong mainly to the $08,08.5,09$, and $09.5 \mathrm{~V}$ spectral types (hereinafter, we refer to them simply as O8-9V). In order to carry out our investigation, we initially selected a sample of objects belonging to these classes from the Galactic O Star Catalog (Maì-Apellániz et al. 2004; hereinafter the GOS Catalog). Various stars were previously observed by our group (at optical wavelengths), and we have retrieved the spectra of some others from publicly available archives. After a first examination, we neglected all those objects that presented a peculiar spectrum. This included, for example, stars known to be binaries (having contamination or known to show strong variability in the spectrum) and ON8-9V objects. Due to our aim to study wind lines, we drastically decreased the number of objects by considering only those having both UV and far-UV data. Furthermore, we have also neglected the stars in common with the work of Martins et al. (2005a), since they were analyzed with the same atmosphere code utilized here (CMFGEN). In the end, we have chosen a subsample of five objects, listed in Table 1.

\subsection{Optical data}

For the optical, we used data collected with the MPI $2.2 \mathrm{~m}$ telescope, located at the European Southern Observatory (ESO), in La Silla, Chile. The high resolution $(R=48000)$ FEROS spectrograph was used and the total wavelength coverage is 4000-9000 $\AA$. The spectra were wavelength calibrated and optimally extracted using the available pipeline (for more details see Kaufer et al. 1999).

We also used spectra obtained with the $1.93 \mathrm{~m}$ telescope at the Observatoire de Haute-Provence, France, using ELODIE. This spectrograph has a resolving power $R=42000$ in the wavelength range $\sim 3900-6800 \AA$. The exposure times were set to yield a signal-to-noise $(\mathrm{S} / \mathrm{N})$ ratio above 100 at about $5000 \AA$. The data reduction, the order localization, background estimate/subtraction, and wavelength calibration, were performed 
Table 1. Observational data of the program stars.

\begin{tabular}{|c|c|c|c|c|c|c|c|c|}
\hline \multirow[b]{2}{*}{ star } & \multirow[b]{2}{*}{ spectral type $^{a}$} & \multirow[b]{2}{*}{$V$} & \multicolumn{2}{|c|}{ Far-UV } & \multicolumn{2}{|c|}{ UV } & \multicolumn{2}{|c|}{ Optical } \\
\hline & & & ${\text { data } \operatorname{set}^{b}}^{b}$ & date & data set & date & instrument & date \\
\hline HD 216898 & O9IV, O8.5V & 8.04 & F-A0510303 & $2000-08-03$ & SWP43934 & 1992-02-05 & $\overline{\mathrm{OHP} / \mathrm{ELODIE}}$ & $2004-08-29$ \\
\hline HD 326329 & O9V & 8.76 & F-B0250501 & 2001-08-09 & WP48698 & $1993-0$ & ESO/FEROS & $2005-06-25$ \\
\hline HD 66 & $\mathrm{O} 8 \mathrm{~V}, \mathrm{O} 9 \mathrm{~V}$ & 9.43 & F-P1011801 & 2000-04-06 & WP49080 & 1993-11-03 & ESO/FEROS & $2004-12-22$ \\
\hline$\zeta \mathrm{Oph}$ & O9.5Vnn & 2.56 & C-C002 & $1972-08-29^{\dagger}$ & SWP36162 & 1989-04-29 & OHP/ELODIE & 1998-06-11 \\
\hline HD 216532 & $08.5 \mathrm{~V}((\mathrm{n}))$ & 8.03 & F-A0510202 & 2000-08-02 & SWP34226 & 1988-09-11 & OHP/ELODIE & $2005-11-10$ \\
\hline
\end{tabular}

${ }^{a}$ Spectral types come from Lesh (1968), Schild et al. (1969), Garrison (1970), Walborn (1973), and MacConnell \& Bidelman (1976).

${ }^{b}$ Satellite used to obtain spectra: $\mathrm{F}=$ FUSE and $\mathrm{C}=$ Copernicus.

$\dagger$ Orbits \#115-247 (Snow et al. 1977).

using the available pipeline (see Baranne et al. 1996). Each order was normalized by a polynomial fit to the continuum, specified by carefully selected continuum windows. Finally we merged the successive orders to reconstruct the full spectrum. The spectrum of $\zeta$ Oph was obtained from the ELODIE archive (see Moultaka et al. 2004, for more details). We have retrieved small portions of its pipeline-treated spectrum and then normalized them using polynomial fits.

\subsection{Ultraviolet data}

Different sources were used for the UV and far-UV data. We have retrieved spectra from the IUE and FUSE satellites using the Multimission Archive at $\mathrm{STScI}^{2}$. For the IUE data, the high resolution SWP mode was preferred $(\sim 0.2 \AA)$, but spectra in the LWP/LWR region were also used to help in the determination of reddening parameters $(R$ and $E(B-V))$. For the FUSE data we have relied only on the LiF2A channel, which comprises the $\sim 1087-1182 \AA$ interval. Spectra of the same object were co-added and smoothed with a five point average for better signal-to-noise $(\mathrm{S} / \mathrm{N})$ ratio and clarity. Although small, the $\mathrm{LiF} 2 \mathrm{~A}$ region provided two very useful features for our study, namely, P V $\lambda \lambda 1118,1128$ and C III $\lambda 1176$ (see Sect. 7.1). We have avoided other FUSE channels covering wavelengths less than $\sim 1087 \AA$ due to severe interstellar contamination. For one object of our sample, $\zeta$ Oph, there is no FUSE data available. Therefore, we have used far-UV data obtained with the Copernicus ("OAO-3") satellite. The spectrum was retrieved from the VizieR Service ${ }^{3}$ and normalized "by eye".

\section{Models and assumptions}

In order to obtain the stellar and wind parameters of the stars of our sample we used the atmosphere codes TLUSTY (Hubeny \& Lanz 1995) and CMFGEN (Hillier \& Miller 1998). The TLUSTY code assumes a plane-parallel geometry, hydrostatic and radiative equilibrium, non-LTE, includes line-blanketing, and can only model lines that are formed in the photosphere. On the other hand, the radiative transfer and statistical equilibrium equations in CMFGEN are solved for a spherically symmetric outflow. The effect of line-blanketing is also taken into account, via a super-level formalism (for more details see Hillier \& Miller 1998). For the outflow we assume a $\beta$ velocity law which connects smoothly (at depth) with a hydrostatic structure provided by TLUSTY.

\footnotetext{
${ }^{2}$ MAST - http://archive.stsci.edu/

${ }^{3}$ http://webviz.u-strasbg.fr/viz-bin/VizieR
}

\subsection{Methodology}

To start our analysis, we used a new grid of TLUSTY model atmospheres based on the OSTAR2002 grid (Lanz \& Hubeny 2003). The new grid uses finer sampling steps in effective temperature $(1000 \mathrm{~K})$ and surface gravity $(0.2 \mathrm{dex})$, updated model atoms (for $\mathrm{Ne}$ and $\mathrm{S}$ ions, C III, N II, N IV, O II, and O III), and additional ions (Mg II, Al II, Al III, Si II, and Fe II; see Lanz \& Hubeny 2007). In order to derive $T_{\text {eff }}$, we used mainly He I $\lambda 4471$ and He II $\lambda 4542$ as diagnostic lines, but fits to other transitions such as He I $\lambda 4713$ and He II $\lambda 4686$ were also checked for consistency. Typical errors for $T_{\text {eff }}$ range from 1 to $2 \mathrm{kK}$. Our derivation of $\log g$ was based on the fit to the $\mathrm{H} \gamma$ wings. For this parameter, the uncertainty varies from 0.1 to 0.2 dex, depending on the object. The rotational velocities $(v \sin i)$ were adopted from previous studies (e.g., Penny 1996; Howarth et al. 1997) and/or refined/estimated from the fitting process when necessary. After we derived the basic photospheric properties (i.e., $v \sin i, \log g$, and $T_{\text {eff }}$ ) we switched to CMFGEN to continue our investigation. As in previous studies, we noted a good agreement between TLUSTY and CMFGEN (e.g., Bouret et al. 2003). In general, conspicuous discrepancies were resolved when we increased the number of species or atomic levels and/or super-levels in CMFGEN.

Once effective temperatures were obtained, we computed the radii by adopting luminosities typical of the 08-O9 dwarfs (see Table 4 of Martins et al. 2005b), following the equation $R_{\star}=\left(L_{\star} / 4 \pi \sigma T_{\mathrm{eff}}^{4}\right)^{1 / 2}$. As we did not normalize the UV spectra, we used the reddening parameters $R$ and $E(B-V)$ (following Cardelli et al. 1988) and the distance as free parameters to match the continuum in the IUE region. When a good fit was achieved, the $R, E(B-V)$, and the distance used were considered representatives for the object in question. The values obtained were generally consistent with the ones estimated in the literature (see Sect. 4). Whenever a large discrepancy was encountered, we revised our adopted $L_{\star}$ and hence $R_{\star}$, keeping $T_{\text {eff }}$ constant. Generally, reasonable agreement was found between the final models and the observed absolute fluxes in the UV. In some cases, however, local scalings of the theoretical continuum had to be made.

The two main wind parameters to be determined are the mass-loss rate and the terminal velocity. Ideally, the determination of the mass-loss rates should be done by fitting P-Cygni profiles and emission-line profiles in the optical (e.g., $\mathrm{H} \alpha$ ) and in the UV (e.g., C IV $\lambda \lambda 1548,1551, \mathrm{~N}$ V $\lambda \lambda 1239,1243$, Si IV $\lambda \lambda 1394,1403)$. In the case of late-type O dwarfs, however, most of these wind transitions are absent and the best estimator of $\dot{M}$ is C IV $\lambda \lambda 1548,1551$. For the velocity structure we have assumed $\beta=1$ for all our models. Tests with $\beta$ ranging from 0.8 to 1.6 did not improve the quality of the C IV $\lambda \lambda 1548,1551$ fits. The terminal velocity $\left(v_{\infty}\right)$ is a very difficult parameter to 
Table 2. Atomic data used in the CMFGEN models.

\begin{tabular}{l|cccc}
\hline \hline Ion & \multicolumn{2}{|c}{ Basic models } & \multicolumn{2}{c}{ Full models } \\
& \# levels & \# super-levels & \# levels & \# super levels \\
\hline H I & 30 & 30 & 30 & 30 \\
He I & 69 & 69 & 69 & 69 \\
He II & 30 & 30 & 30 & 30 \\
C II & 22 & 22 & 338 & 104 \\
C III & 243 & 99 & 243 & 217 \\
C IV & 64 & 64 & 64 & 64 \\
N III & 287 & 57 & 287 & 57 \\
N IV & 70 & 44 & 70 & 44 \\
N V & 49 & 41 & 49 & 41 \\
O II & 296 & 53 & 340 & 137 \\
O III & 115 & 79 & 115 & 79 \\
O IV & 72 & 53 & 72 & 53 \\
Ne II & - & - & 242 & 42 \\
Mg II & - & - & 65 & 22 \\
Al III & - & - & 65 & 21 \\
Si III & - & - & 45 & 25 \\
Si IV & 33 & 22 & 33 & 22 \\
P V & 62 & 16 & 62 & 16 \\
S IV & - & - & 142 & 51 \\
S V & - & - & 216 & 33 \\
Fe III & - & - & 607 & 65 \\
Fe IV & 1000 & 64 & 1000 & 64 \\
Fe V & 1000 & 45 & 1000 & 45 \\
\hline & & & &
\end{tabular}

obtain for O8-O9V stars, since there are no saturated P-Cygni profiles. We initially used literature estimates for $v_{\infty}$, and then modified its value to improve the fits. When estimates were not available, we started our models assuming $v_{\infty}$ values between $1300-1700 \mathrm{~km} \mathrm{~s}^{-1}$. We conservatively assume the uncertainty in this parameter to be about $500 \mathrm{~km} \mathrm{~s}^{-1}$. For the mass-loss rate, we adopt a conservative uncertainty of $\sim 0.7 \mathrm{dex}$, following the analysis performed by Martins et al. (2005a).

We assumed a fixed microturbulence $\xi_{t}$ of $15 \mathrm{~km} \mathrm{~s}^{-1}$ in the co-moving computation of our models. However, for computation of the spectra the code (CMF_FLUX) allows for a depth dependent microturbulent velocity, according to the equation $\xi(r)=\xi_{\min }+\left(\xi_{\max }-\xi_{\min }\right) v(r) / v_{\infty}$. We fixed $\xi_{\min }$ at $5 \mathrm{~km} \mathrm{~s}^{-1}$ (near the photosphere) and for $\xi_{\max }$ we assumed a value of $0.1 v_{\infty}$. As already reported in previous studies, slightly different values for these parameters do not cause significant changes in the spectrum.

We used two sets of atomic data for CMFGEN in our study. Initial fits to the observed spectra, tests, and the exploration of higher mass-loss rates (Sect. 7) were done using models with a reduced number of species and levels/super-levels (basic models). For our final model for each object, a more complete set of atomic data was used (full). In total, 4.5GB (2GB) of memory space (RAM) was required to compute each full (basic) model. The details of the atomic data are shown in Table 2. This approach saved a lot of computational time and resources, without compromising the results. The main difference between these two sets of data is the quality of the optical fit. Some lines could only be reproduced after the inclusion of additional species/ions (e.g., Mg II $\lambda 4481$ and Si III $\lambda 4553$ ) or by increasing the number of levels and/or super-levels. In the ultraviolet, only the 1850-2000 $\AA$ region is affected by the larger data set (being better fitted), due to the inclusion of Fe III. In other parts of the spectrum the changes are minor. A solar chemical abundance was adopted for all elements (following Grevesse \& Sauval 1998). As shown later in Sect. 4, it provides reasonable fits to the observations. In this paper, only the abundance of carbon is investigated in detail (see Sect. 6), given its direct relation with the weak wind problem through C IV $\lambda \lambda 1548,1551$.

\subsection{Clumping and $X$-rays}

Currently, the effect of wind density inhomogeneities (i.e., clumping) is implemented in CMFGEN through a depth dependent volume filling factor $f=f_{\infty}+\left(1-f_{\infty}\right) \mathrm{e}^{-v(r) / v_{\mathrm{cl}}}$. The parameter $v_{\mathrm{cl}}$ determines the velocity at which clumping starts to be important. In the photosphere the model is homogeneous while for velocities higher than $v_{\mathrm{cl}}, f$ converges quickly to $f_{\infty}$. Because of the lack of wind lines in the spectra of the stars studied here, we chose to not use clumping in our models and set $f_{\infty}=1$. The use of clumping usually requires a decrease in the massloss rate of a (previously homogeneous final) model by a factor of $1 / \sqrt{f_{\infty}}$, in order to fit (again) the observed spectra. Thus, the mass-loss in our final models may be overestimated by a factor of about three if $f_{\infty} \sim 0.1$ in the wind of these objects.

CMFGEN also allows for Auger ionization by X-rays. The $\mathrm{X}$-rays are thought to arise from shocks generated by wind instabilities. The X-rays are distributed throughout the wind, with the X-ray emissivity primarily controlled by a shock temperature plus a filling factor parameter (see Hillier \& Miller 1998, for details). The effects of X-rays in the atmosphere of O dwarfs were discussed in detail by Martins et al. (2005a). These authors convincingly show that X-rays are not as important for early-type as they are for late-type O stars (see also MacFarlane et al. 1994). For early-type objects (denser winds), the ionization and wind lines such as C IV $\lambda \lambda 1548,1551$ do not present significant changes when $\mathrm{X}$-rays are included. Only wind lines belonging to super-ions (O VI $\lambda \lambda 1032,1038$ and to a lesser extent, N V $\lambda \lambda 1239,1243$ ) are affected. In contrast, for late-type stars it is observed that the ionization structure and the C IV $\lambda \lambda 1548,1551$ line changes considerably. Because the ionization is shifted to $\mathrm{C}^{4+}$ and $\mathrm{C}^{5+}$, mass-loss rates about ten times larger are sometimes required to fit the observed C IV $\lambda \lambda 1548,1551$ line. Thus higher mass-loss rates are favored when X-rays are included. In this paper, we use a fixed shock temperature of $3 \times 10^{6} \mathrm{~K}$ and the filling factor is chosen to keep $\log L_{\mathrm{x}} / L_{\mathrm{Bol}}$ close to -7.0 (within \pm 0.1 ). We recall that a $\log L_{\mathrm{x}} / L_{\mathrm{Bol}}$ of about -7.0 is the typical value observed (see e.g., Sana et al. 2006). For HD 326329 however, we used models with a higher ratio, in accord with the observations $\left(\log L_{\mathrm{X}} / L_{\mathrm{Bol}} \sim-6.5\right.$; Sana et al. 2006). For $\zeta$ Oph, we used $\log L_{\mathrm{X}} / L_{\mathrm{Bol}} \sim-7.3$ (Oskinova 2005; Oskinova et al. 2006).

\section{Spectral analysis}

In this section we present the analysis of our sample. A brief introduction about each object is made, followed by the presentation of our CMFGEN model fits to the UV and optical observed spectra. The discrepancies found are discussed and a comparison to previous results in the literature is given when necessary.

\section{1. $H D 216898$}

HD 216898 belongs to the Cepheus OB3 association (Garmany $\&$ Stencel 1992). No unified and line-blanketed model atmospheres have been used to analyze this object. Its mass-loss rate was investigated two decades ago by Leitherer (1988), who could only establish an upper $\operatorname{limit}(\log \dot{M}<-6.97)$ from the $\mathrm{H} \alpha$ profile. 
Our fits to the far-UV and UV spectra are presented in Fig. A.1. The $E(B-V)$ and distance used are 0.7 and $1.1 \mathrm{kpc}$, respectively. Four intense transitions can be noted in the FUSE region: P V $\lambda \lambda 1118,1128$, Si IV $\lambda \lambda 1122,1128$, and C III $\lambda 1176$. The various narrow deep absorptions that are not reproduced by our models are of interstellar origin. As can be seen in Fig. A.1 (bottom), our final model also fits correctly the main features of the IUE spectrum. In the 1380-1590 $\AA$ interval, Si IV $\lambda \lambda 1394,1403$ and C IV $\lambda \lambda 1548,1551$ are the most evident transitions, while most of the other absorptions can be attributed to Fe IV lines (an iron forest). A TLUSTY model, with the same photospheric parameters as used in CMFGEN, presents a similar fit to the far-UV and UV, with the exception of C IV $\lambda \lambda 1548,1551$. In TLUSTY, this doublet is seen as two well separated absorptions, while the observations show an extended blue feature that is only reproduced with CMFGEN (i.e., C IV $\lambda \lambda 1548,1551$ is formed in the wind).

The mass-loss rate needed to fit C IV $\lambda \lambda 1548,1551$ is $\log \dot{M}=-9.35$. If we take the other physical parameters derived for this object $\left(T_{\text {eff }}, L_{\star}\right.$, and $\left.M_{\star}=g R_{\star}^{2} / G\right)$ and use the mass-loss rate recipe of Vink et al. (2000), we derive a theoretical mass-loss rate of $\log \dot{M}_{\text {Vink }}=-7.22$, which is more than two orders of magnitude larger than the observed mass-loss rate. If we use mass-loss rates higher than that adopted for our final model, a deeper blue absorption (with respect to the line center) and an intense emission starts to be seen (i.e., a more normal P-Cygni profile is developed), contrary to what is observed. On the other hand, lower $\dot{M}$ 's slowly approach the photospheric prediction by TLUSTY, as expected.

In Fig. A.2 we present our fit to the optical spectrum of HD 216898. The five different spectral regions shown contain the diagnostic lines used to derive the photospheric parameters (see Sect. 3.1). The effective temperature could be very well constrained. The $\mathrm{He}$ I $\lambda 4471 / \mathrm{He}$ II $\lambda 4542$ and He I $\lambda 4713 / \mathrm{He}$ II $\lambda 4686$ ratios are well reproduced with a $T_{\text {eff }}=(34 \pm 1) \mathrm{kK} . \mathrm{H} \gamma$ has a reasonable fit but the model is somewhat stronger than the observed line. The same happens to $\mathrm{H} \alpha$. Nevertheless, their wings are well matched with a $\log g$ of $4.0 \pm 0.1$. Details regarding this last transition are discussed later in Sect. 7.2. Some of the most intense lines in Fig. A.2 are: He I $\lambda 4026, \mathrm{H} \delta, \mathrm{H} \gamma, \mathrm{He}$ I $\lambda 4388, \mathrm{He}$ I $\lambda 4471, \mathrm{He}$ II $\lambda 4542$, C III $\lambda 4647$, and C III $\lambda 4650$ (with small contribution of O II), He II $\lambda 4686, \mathrm{He}$ I $\lambda 4713$, and $\mathrm{H} \alpha$. These same transitions are easily identified in the other objects of our sample, and in other typical 08-9V stars, that have $v \sin i$ less than about $100 \mathrm{~km} \mathrm{~s}^{-1}$. For rapid rotators such as $\zeta$ Oph and HD 216532, several profiles are broadened/blended and in some cases we cannot distinguish individual transitions. The $\mathrm{H} \alpha$ observed in HD 216898 is symmetric. Indeed, all model predictions yield symmetrical profiles for $\mathrm{H} \alpha$. However, as shown later, some objects present an asymmetric $\mathrm{H} \alpha$ profile of unknown origin.

\subsection{HD 326329}

HD 326329 is located in the NGC 6231 cluster, at the nucleus of the Sco OB1 association. It belongs to a resolved triple system $(\mathrm{O} 9 \mathrm{~V}+\mathrm{O} 9 \mathrm{~V}+\mathrm{B} 0 \mathrm{~V}$; GOS Catalog), but it is probably a single star itself since it does not show photometric or spectroscopic variations (Garcia \& Mermilliod 2001). So far, no atmosphere models have been used to analyze its spectrum.

In Fig. A.3 we present our final model for the far-UV and UV observed spectra. From the IUE continua, we derived an $E(B-V)$ value of 0.44 and a distance of $1.99 \mathrm{kpc}$, in agreement with the study of the NGC 6231 cluster made by Baume et al. (1999). As for the case of HD 216898, good agreement is found in the FUSE spectral region. The P V $\lambda \lambda 1118,1128$, Si IV $\lambda \lambda 1122,1128$, and C III $\lambda 1176$ lines are well reproduced. The IUE spectrum, however, could not be entirely matched; the observed C IV $\lambda \lambda 1548,1551$ is considerably deeper than in the model. We have tried several different tests to fix this discrepancy (e.g., changes in the $\beta$ velocity law, lower terminal velocities, increased turbulence), but they all turned out to be unsuccessful. Although there is only one IUE high resolution spectrum for HD 326329 (shown in Fig. A.3), model comparisons to the few available low-resolution IUE spectra confirm the too deep/broad feature. In the study of Martins et al. (2005a) no such problem was found; the $\mathrm{O} 9$ dwarfs analyzed in their study show C IV $\lambda \lambda 1548,1551$ profiles that are well reproduced by the models. Interestingly, one more star of our sample also shows a profile deeper than the model, namely, HD 216532 (Sect. 4.5).

Despite the problem described above, we can still be confident about the mass-loss rate chosen for HD 326329, $\log \dot{M}=$ -9.22 , within \pm 0.7 dex. First, there is a lack of emission in the observed C IV $\lambda \lambda 1548,1551$ P-Cygni profile, suggesting that $\dot{M}$ must indeed be low. Further, when higher values for $\dot{M}$ are used, a synthetic line with an intense (unobserved) emission and discrepant blue absorption appears (see Fig. A.3; dotted line). On the other hand, when lower mass-loss rates are used, C IV $\lambda \lambda 1548,1551$ slowly turns into photospheric features like those predicted by TLUSTY.

Our fit to the optical spectrum of HD 326329 is presented in Fig. A.4. The He I and He II lines are well reproduced with a $T_{\text {eff }}=(31 \pm 1) \mathrm{kK}$. The fit to the $\mathrm{H} \gamma$ wings gives a surface gravity $(\log g)$ of $3.9 \pm 0.1$. Both $T_{\text {eff }}$ and $\log g$ were previously estimated by Mathys et al. (2002) who found $31700 \mathrm{~K}$ and 4.6, respectively, by using $u v b y \beta$ photometry. Although our derived temperature is compatible with their value, their higher $\log g$ is not supported by our analysis. In fact, a $\log g$ much higher than 4.0 is not expected for O stars (see e.g., Vacca et al. 1996; Martins et al. 2005b). Although a fairly good agreement is found in several parts of the optical spectrum, we could not achieve a good fit to $\mathrm{H} \alpha$. This line is weaker than in the model and is also asymmetric. Its blue wing has a "bump" which distorts the profile. This is not seen in the other hydrogen lines. We have not used $\mathrm{H} \alpha$ to derive any stellar or wind parameters of the objects of our sample. However, we later discuss the effects that different mass-loss rates have on this line (Sect. 7.2).

\section{3. $H D 66788$}

Figure A.5 shows our fits to the ultraviolet spectrum of HD 66788. The C IV $\lambda \lambda 1548,1551$ feature is reproduced with a mass-loss rate of $\log \dot{M}=-8.92$. This value is much lower than the one predicted, $\log \dot{M}_{\mathrm{Vink}}=-6.95$. The reddening and distance derived are $E(B-V)=0.22$ and $d=4.8 \mathrm{kpc}$. Although large, this distance is consistent with estimates encountered in the literature (Reed 1993; Kaltcheva \& Hilditch 2000).

In Fig. A.6 we show our fit to the optical spectrum. The features found in HD 216898 and HD 66788 are very similar, suggesting similar photospheric parameters (see Sect. 4.1). Indeed, from our fits to the $\mathrm{H} \gamma$ we derive a $\log g=4.0 \pm 0.1$, and from the He I $\lambda 4471$ and He II $\lambda 4542$ lines we obtain a $T_{\text {eff }}=(34 \pm 1) \mathrm{kK}$, as in HD 216898. Their rotational velocities are also very similar, about $60 \mathrm{~km} \mathrm{~s}^{-1}$. We note that the $\mathrm{H} \alpha$ line in HD 66788 is slightly asymmetric. Although the overall agreement is reasonable, its blue wing has a weak absorption not predicted by the model. 


\section{4. $\zeta O p h$}

$\zeta$ Oph (also known as HD 149757) is a well studied runaway star which presents several interesting characteristics. It has been known for a long time to present different kinds of spectral variability, such as discrete absorption components in the UV (DACs), emission line episodes, and line profile variations (LPVs) (see e.g., Howarth et al. 1984; Reid et al. 1993; Howarth et al. 1993; Jankov et al. 2000; Walker et al. 2005). This object also shows a very high rotational velocity: $v \sin i$ values around 400 or even $500 \mathrm{~km} \mathrm{~s}^{-1}$ have been reported in the literature (Walker et al. 1979; Repolust et al. 2004).

A reason to include $\zeta$ Oph in our sample is that it is considered to have an $\dot{M}$ estimate based on $\mathrm{H} \alpha$ which is in good agreement with the predictions of the radiative wind theory (Mokiem et al. 2005, 2007). Thus, it is interesting to check if a fit from the UV to the optical (including $\mathrm{H} \alpha$ ) can be achieved with CMFGEN using a low $\dot{M}$. We stress however, that the very high rotational velocity presented by $\zeta$ Oph makes analyses more difficult. First, fast rotation can distort the shape and induce temperature and gravity variations of the stellar surface (see e.g., Frémat et al. 2005). Furthermore, rotation might lead to a wind which is not spherically symmetric. In such cases, 1D unified atmosphere models should be considered as an approximation. Second, the analysis of the diagnostic lines is more difficult than for the case of slower rotators because features in the spectrum are broadened and more likely to be blended.

Our final model and the IUE spectrum of $\zeta$ Oph are shown in Fig. A.7. A distance of $146 \mathrm{pc}$ and an $E(B-V)=0.36$ were used for the continuum fit. Due to the high $v \sin i$ involved, the photospheric iron forest is considerably broadened. Despite its weakness, $\mathrm{N} v \lambda \lambda 1239,1243$ can be easily distinguished in the spectrum, as well as the C III $\lambda 1176$ and the Si IV $\lambda \lambda 1394,1403$ lines. While the model can successfully reproduce the main UV features observed, some discrepancies can be noted. The C IV $\lambda \lambda 1548,1551$ line cannot be matched in detail; the synthetic line is slightly more intense and narrower than that observed. A model with a lower mass-loss rate does not solve the problem, and additionally makes the fit to $\mathrm{N} v \lambda \lambda 1239,1243$ worse. On the other hand, higher mass-loss rate models yield both stronger emission and blue absorption in the P-Cygni profile, in contrast with the observations (see Fig. A.7; dotted line). The mass-loss rate used, $\log \dot{M}=-8.80$, should be certain within a factor of three (i.e., $\sim 0.5 \mathrm{dex}$ ). This value is much lower than that derived in the study of Mokiem et al. (2005). Using the FASTWIND code, and based on $\mathrm{H} \alpha$, these authors derive $\log \dot{M}(\mathrm{H} \alpha)=-6.85$. Our value is also much lower than the theoretical value predicted by the theoretical recipe of Vink et al. (2000), $\log M_{\text {Vink }}=-6.89$. We come back to this question later in Sect. 7. Other smaller discrepancies can also be seen in the Si IV $\lambda \lambda 1394,1403$ line fit and in the $\sim 1550-1650 \AA$ interval. Despite our efforts, they could not be resolved. Due to the very high rotational velocity presented by $\zeta$ Oph, future studies using $2 \mathrm{D}$ atmosphere models will be useful to address some of these problems.

In Fig. A.8 we present our model fit to the optical spectrum. As we mentioned earlier, a high rotational velocity convolution is necessary to fit the broad features observed $(v \sin i \sim$ $400 \mathrm{~km} \mathrm{~s}^{-1}$ ). Despite the high rotation, the effective temperature and surface gravity could be derived, but with a higher uncertainty than in the other objects of our sample. From the He I and He II lines, we have obtained a $T_{\text {eff }}=(32 \pm 2) \mathrm{kK}$. From the $\mathrm{H} \gamma$ profile we estimate a low surface gravity compared to the other objects: $\log g=3.6 \pm 0.2$. This value should be regarded as an effective gravity - the surface gravity attenuated by rotation. A "centrifugal correction" can be applied in order to obtain the true surface gravity, according to the equation $\log g_{\text {true }}=$ $\log \left(g_{\text {eff }}+v \sin i^{2} / R_{\star}\right)$ (Repolust et al. 2004). For $\zeta$ Oph, we find a $\log g_{\text {true }}$ of $\sim 3.8$. This correction however, neglects any distortion in the stellar shape. A treatment allowing the oblateness of the star was presented by Howarth \& Smith (2001) for three fast rotators, including $\zeta$ Oph. These authors derived a polar (equatorial) gravity of $\sim 4.0(\sim 3.6)$, a polar (equatorial) radius of $7.5 R_{\odot}$ $\left(9.1 R_{\odot}\right)$, and an effective temperature of $\sim 34 \mathrm{kK}$. The radius obtained from our $T_{\text {eff }}$ and $L_{\star}$ is $9.2 R_{\odot}$. Therefore, our stellar parameters for $\zeta$ Oph are compatible with the equatorial values found by Howarth \& Smith (2001). They also show agreement with other works in the literature (Repolust et al. 2004; Mokiem et al. 2005; Villamariz \& Herrero 2005).

$\mathrm{H} \alpha$ and a few others lines have been observed to show emission episodes (in the form of double peaks) on different occasions (see e.g., Niemela \& Mendez 1974; Ebbets 1981). In general, however, $\mathrm{H} \alpha$ shows a symmetric absorption profile, with an equivalent width $\left(W_{\lambda}\right)$ of $(2.7 \pm 0.4) \AA$, and with a central depth of about 0.8 of the continuum intensity. These characteristics can be considered to represent $\zeta$ Oph's quiescent spectrum (see Reid et al. 1993, and references therein). From our spectrum we measure a $W_{\lambda}$ of $(3.0 \pm 0.3) \AA$ and the deepest part of the absorption is at $\sim 0.8$ of the continuum level, confirming a quiescent-like spectrum. The profile, however, presents narrower wings than in our final model (see Fig. A.8, bottom). This problem can also be perceived in the model presented by Mokiem et al. (2005) calculated using the FASTWIND code (see their Fig. 12). A decrease in the $v \sin i$ to $350 \mathrm{~km} \mathrm{~s}^{-1}$ improves the fit to the wings, but the line center becomes slightly deeper than observed. A lower $v \sin i$ also has a negative effect on other optical lines such as $\mathrm{H} \delta$ and $\mathrm{H} \gamma$.

\subsection{HD 216532}

HD 216532 is a relatively fast rotator which belongs to the Cepheus OB3 association. According to Howarth et al. (1997) its $v \sin i$ is $\sim 190 \mathrm{~km} \mathrm{~s}^{-1}$, a value that we confirm from our model fits. Leitherer (1988) estimated its stellar parameters and derived an upper limit for the mass-loss rate of $\log \dot{M}<-7.08$ from $\mathrm{H} \alpha$. The present study is the first to analyze quantitatively its far-UV to optical spectra.

Our fit to the far-UV and UV data is presented in Fig. A.9. For the $E(B-V)$ and the distance, we inferred 0.72 and $1.15 \mathrm{kpc}$, respectively. In the FUSE region, although the overall agreement is fair, some discrepancies can be noted. The observed P V $\lambda \lambda 1118,1128$ and Si IV $\lambda \lambda 1122,1128$ lines are deeper than in the model. Although the synthetic continuum is somewhat higher than the observed, the problem remains if we normalize the spectrum locally. As in HD 326329, we could not fit the C IV $\lambda \lambda 1548,1551$ doublet in the IUE spectrum. Compared to the profiles found in HD 216898 and HD 66788, the C IV $\lambda \lambda 1548,1551$ doublet in HD 216532 is broader and deeper. Nevertheless, the mass-loss rate of our final model, $\log \dot{M}=-9.22$, should be certain within about \pm 0.4 dex. Higher values result in P-Cygni profiles whose emissions are too strong, and this is not observed. An example is shown in Fig. A.9. As already discussed, lowering the mass-loss rate slowly changes the C IV $\lambda \lambda 1548,1551$ feature into two photospheric absorptions.

In Fig. A.10 we present the observed optical spectrum of HD 216532 along with our final model. Because of the relatively high $v \sin i$ of this object, $\sim 190 \mathrm{~km} \mathrm{~s}^{-1}$, the lines are broader than 
Table 3. Stellar and wind parameters.

\begin{tabular}{l|ccccc}
\hline \hline Star & HD 216898 & HD 326329 & HD 66788 & $\zeta$ Oph & HD 216532 \\
\hline Spec. type & O9IV, O8.5V & O9V & O8-9V & O9.5Vnn & O8.5V $((\mathrm{n}))$ \\
$\log g$ & $4.0 \pm 0.1$ & $3.9 \pm 0.1$ & $4.0 \pm 0.1$ & $3.6 \pm 0.2$ & $3.7 \pm 0.2$ \\
$T_{\text {eff }}(\mathrm{kK})$ & $34 \pm 1$ & $31 \pm 1$ & $34 \pm 1$ & $32 \pm 2$ & $33 \pm 2$ \\
$v \sin i\left(\mathrm{~km} \mathrm{~s}^{-1}\right)$ & 60 & 80 & 55 & 400 & 190 \\
$\log L / L_{\odot}$ & $4.73 \pm 0.25$ & $4.74 \pm 0.10$ & $4.96 \pm 0.25$ & $4.86 \pm 0.10$ & $4.79 \pm 0.25$ \\
$R_{\star}\left(R_{\odot}\right)$ & $6.7_{-1.7}^{+2.3}$ & $8.0_{-1.0}^{+1.1}$ & $8.7_{-2.2}^{+3.0}$ & $9.2_{-1.4}^{+1.7}$ & $7.5_{-2.0}^{+2.7}$ \\
$M_{\star}\left(M_{\odot}\right)$ & $17_{-8}^{+15}$ & $19_{-6}^{+8}$ & $26_{-12}^{+23}$ & $13_{-6}^{+10}$ & $12_{-6}^{+14}$ \\
$v_{\infty}\left(\mathrm{km} \mathrm{s}^{-1}\right)$ & $1700 \pm 500$ & $1700 \pm 500$ & $2200 \pm 500$ & $1500 \pm 500$ & $1500 \pm 500$ \\
$\log \dot{M}$ & $-9.35 \pm 0.7$ & $-9.22 \pm 0.7$ & $-8.92 \pm 0.7$ & $-8.80 \pm 0.7$ & $-9.22 \pm 0.7$ \\
$\log \dot{M}_{\text {Vink }}$ & -7.22 & -7.38 & -6.95 & -6.89 & -6.92 \\
\hline $\log L_{\mathrm{x}} / L_{\mathrm{Bol}}$ & -7.00 & -6.69 & -7.06 & -7.31 & -7.00 \\
$\log L / c^{2}$ & -8.42 & -8.41 & -8.19 & -8.29 & -8.36 \\
$E(B-V)$ & 0.7 & 0.44 & 0.22 & 0.36 & 0.72 \\
$R$ & 3.5 & 3.1 & 2.8 & 2.9 & 3.5 \\
$\operatorname{distance}(\mathrm{pc})$ & 1100 & 1990 & 4800 & 146 & 1150 \\
\hline
\end{tabular}

those in HD 216898, HD 326329, and HD 66788. With an effective temperature of $\sim 33 \mathrm{kK}$, we achieve a good match to the observed He I $\lambda 4471$ and He II $\lambda 4542$ lines. To fit the $\mathrm{H} \gamma$ profile, we used $\log g=3.7$.

\section{Analysis of the results}

In this section we summarize the results of our spectral analysis and make a comparison to previous works and theoretical predictions. The stellar and wind parameters obtained are presented in Table 3. For comparison, we also list the theoretical mass-loss rates computed with the recipe of Vink et al. (2000). Additional parameters, such as the reddening and distance, are shown in the lower part of the table. For HD 216898, HD 66788, and HD 216532, we have fixed $\log L_{\mathrm{X}} / L_{\mathrm{Bol}}$ at the canonical value of $\sim-7.0$. For HD 326329 and $\zeta$ Oph, we chose $L_{X} / L_{\text {Bol }}$ ratios close to those recently observed (Oskinova 2005; Oskinova et al. 2006; Sana et al. 2006).

Overall, the stellar properties of our sample are quite homogeneous. The effective temperatures range from $\sim 30$ to $34 \mathrm{kK}$, and the radii are between 6 and $10 R_{\odot}$. The lowest surface gravities are for HD 216532 and $\zeta$ Oph, which is to be expected since both stars are rapid rotators. In their case, the $\log g$ measured should be interpreted as an effective surface gravity. The derived physical properties show a fair agreement with the latest calibration of Galactic O star parameters for the O8-9V spectral types (see Martins et al. 2005b).

In order to analyze the stellar and wind parameters we plot our results in Fig. 1 in two different ways. First, the massloss rates are compared to the theoretical predictions of Vink et al. (2000) and second, the wind parameters are used to construct the modified wind momentum luminosity relation (WLR): $\left(\dot{M} v_{\infty} \sqrt{R_{\star}}\right) \times L_{\star}$. In each of these plots, we include the results of Martins et al. (2005a) for eleven O dwarfs, as well as the data gathered by Mokiem et al. (2007) for O and B dwarfs, giants, and supergiants (see their Table A1). In order to simplify the analysis, and to exclude metallicity effects, only Galactic objects are considered.

In the plots presented in Fig. 1, we perceive the same basic result; the stars of our sample gather around the same places occupied by the four $08-9 \mathrm{~V}$ stars studied in Martins et al. (2005a), namely, $\mu$ Col, AE Aur, HD 46202, and HD 93028. This means that: (i) we have also found a discrepancy of roughly two orders of magnitude between the measured and the predicted mass-loss rates for our program stars; (ii) the modified wind momentum-luminosity relation indeed shows a breakdown or a steepening below $\log L_{\star} / L_{\odot} \sim 5.2$.

The mass-loss rates for the brighter objects, those with $\log L_{\star} / L_{\odot}>5.2$, follow reasonably well the theoretical expectations. The four most luminous early-type dwarfs analyzed in the work of Martins et al. (2005a) have clumped mass-loss rates. Thus, although they fall somewhat below the theoretical line in the $\log \dot{M} \times \log \dot{M}_{\text {Vink }}$ plot and below the fit to the data of Mokiem et al. (2007), they do not present a significant discrepancy if clumping is neglected ${ }^{4}$.

The results derived by us and Mokiem et al. (2005) for the star $\zeta$ Oph are connected by a dashed line in Fig. 1 . The large difference observed is due to the different mass-loss rates derived. While we have $\log \dot{M}=-8.80$ from the C IV $\lambda \lambda 1548,1551$ line, Mokiem et al. (2005) derived $\log \dot{M}=-6.84$ using $\mathrm{H} \alpha$ and the FASTWIND code. The other stellar and wind parameters determined in their study and ours are very similar. The radius, $T_{\text {eff }}$, and $\log L_{\star} / L_{\odot}$ from this (their) paper are 9.2 (8.9) $R_{\odot}$, 32.1 (32) kK, and 4.86 (4.88), respectively. For the wind terminal velocity, we (they) obtain 1500 (1550) $\mathrm{km} \mathrm{s}^{-1}$.

Martins et al. (2005a) also investigated other low luminosity objects $\left(\log L_{\star} / L_{\odot} \lesssim 5.2\right)$. Two of these, HD 93146 and HD 42088, have $\log L_{\star} / L_{\odot}=5.2( \pm 0.2)$ and are classified as $06.5 \mathrm{~V}$ stars. Another object included in their study is HD 152590, an O7.5V star with $\log L_{\star} / L_{\odot} \sim 4.8$. Their massloss rates lie above the $08-9 \mathrm{~V}$ group in the plots in Fig. 1, but still lie below the theoretical predictions. Contrary to the other four earlier $\mathrm{O}$ dwarfs, clumping was not used in their analysis. These three stars suggest that in spectral types $06.5 \mathrm{~V}, \mathrm{O} 7 \mathrm{~V}$, and $07.5 \mathrm{~V}$, a decrease of the wind strength starts to be seen, culminating in very low mass-loss rates for the O8-9V classes.

Also at low luminosities are the following objects analyzed by Mokiem et al. (2005): Cyg OB2\#2 (B1 I), $\tau$ Sco (B0.2V), $10 \mathrm{Lac}$ (O9V), and HD 217086 (O7Vn) (see Fig. 1; filled squares). The quoted mass-loss rates, which were obtained from the $\mathrm{H} \alpha$ line, are in better agreement with the theoretical predictions and thus follow relatively well the WLR. Given these and our UV based results, the origin of the weak wind problem can be thought to reside in the different mass-loss rate diagnostics employed. In fact, Mokiem et al. (2007) have suggested that the problem could be due to uncertainties in the UV method. However, the good agreement of the Mokiem et al. (2005)

\footnotetext{
${ }^{4}$ We recall that the theoretical models of Vink et al. (2000) do not include clumping.
} 

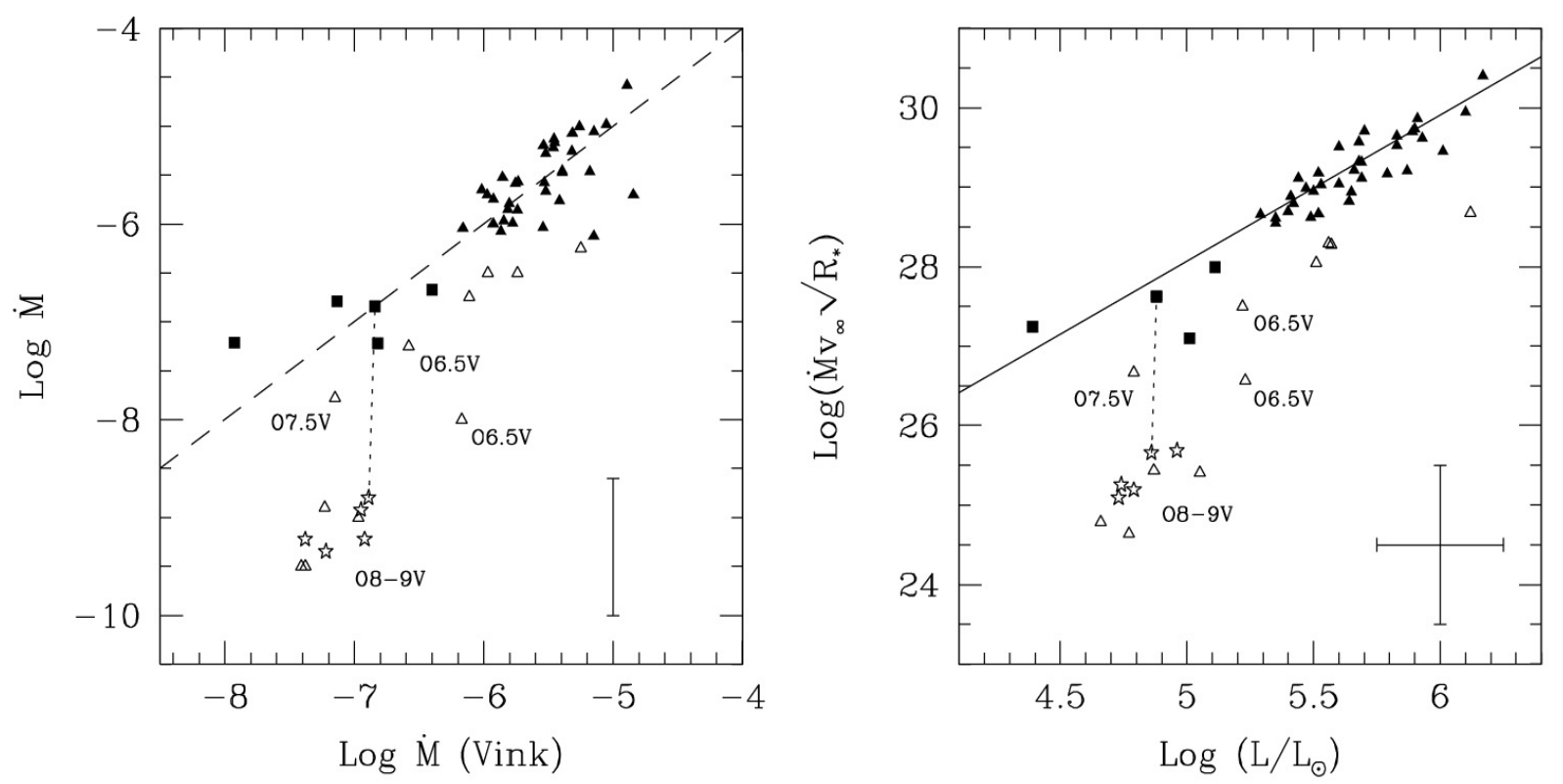

Fig. 1. Comparison of our results to previous results in the literature and theoretical predictions. The objects of our sample are represented by star symbols; the O dwarfs studied by Martins et al. (2005a) by open triangles; the O and B stars compiled by Mokiem et al. (2007) that have $\log L_{\star} / L_{\odot} \lesssim 5.2$ are represented by filled squares and the ones with higher luminosities by filled triangles. Left panel: mass-loss rates compared to theoretical predictions $\left(\dot{M}_{\mathrm{Vink}}\right)$. The dashed line indicates a one-to-one relation. Right panel: modified wind momentum-luminosity relation (WLR). The solid line represents the best fit to the data in Mokiem et al. (2007). The dotted lines link the results obtained in this paper and in Mokiem et al. (2005) for the star $\zeta$ Oph. Low luminosity dwarfs $\left(\log L_{\star} / L_{\odot} \lesssim 5.2\right)$ in this paper and in Martins et al. (2005a) are explicitly indicated by their spectral types (see text for more details).

mass-loss rates with theory is somewhat illusionary - the inferred errors for $\tau$ Sco $(\mathrm{B} 0.2 \mathrm{~V})$ and $10 \mathrm{Lac}(\mathrm{O} 9 \mathrm{~V})$ mean that mass-loss rates an order of magnitude lower are also consistent with the data analyzed.

There are several other O8-9V stars with UV spectra similar to the ones found in our sample. Thus, it is likely that they possess similar wind properties, and thus our analysis would yield the same basic results.

\section{The carbon abundance}

Modeling of the C IV $\lambda \lambda 1548,1551$ profile shows that it is influenced by the carbon abundance $\left(\epsilon_{\mathrm{C}}\right)$, and that by choosing different combinations of $\dot{M}$ and $\epsilon_{\mathrm{C}}$, we can get satisfactory fits to this spectral feature, as long as its optical depth is kept constant at the observed value. This degeneracy is directly apparent from the expression for the Sobolev optical depth, which is proportional to the mass-loss rate, the $\mathrm{C}$ IV ionization fraction $\left(q_{\mathrm{CIV}}\right)$, and the abundance: $\tau_{\mathrm{CIV}} \propto \dot{M} q_{\mathrm{CIV}} \epsilon_{\mathrm{C}}$ (see, for example, Lamers et al. 1999). In the context of the weak wind problem, this relation suggests that we could fit the C IV $\lambda \lambda 1548,1551$ lines using models with mass-loss rates similar to the values predicted by radiative wind theory if we use a low abundance of carbon. However, we are able to reject this hypothesis.

An accurate carbon abundance determination in late O dwarfs is needed to disentangle $\dot{M}$ and $\epsilon_{\mathrm{C}}$. In order to achieve this goal we built a small grid of models with the following $\epsilon_{\mathrm{C}} / \epsilon_{\mathrm{C}_{\odot}}$ ratios: $0.2,0.5,1.0,1.5$, and 3 (in mass fractions). We then analyzed the behavior of the following photospheric transitions: C III $\lambda \lambda 4647-4651, \lambda 1176$, and C IV $\lambda \lambda 5801,5812$. Although these lines do respond to changes in $\epsilon_{\mathrm{C}}$, their sensitivity is limited precluding an accurate determination of the carbon abundance at the present time.
In Fig. 2 we show the fits obtained using models with $\epsilon_{\mathrm{C}}=0.2 \epsilon_{\mathrm{C}_{\odot}}(20 \%$ solar $), \epsilon_{\mathrm{C}}=\epsilon_{\mathrm{C}_{\odot}}($ solar $)$, and $\epsilon_{\mathrm{C}}=3 \epsilon_{\mathrm{C}_{\odot}}$ ( $3 \times$ solar). Although our grid is more extensive, we illustrate only extreme $\epsilon_{\mathrm{C}}$ values, in addition to the solar value, to emphasize the changes. From Fig. 2, we found: (i) with an abundance of $0.2 \epsilon_{\mathrm{C}_{\odot}}$ we cannot fit any of the lines shown; (ii) with 3 times the solar value, we cannot fit the C III $\lambda 1176$ line, because broad wings that are not observed develop in the synthetic spectra; (iii) the 3 times solar abundance is also not supported by C III $\lambda \lambda 4647-4651$ in HD 216898 and HD 66788, and also hints to be too large in HD 326329, $\zeta$ Oph, and HD 216532. From (i) we can conclude that our sample must have a $\epsilon_{\mathrm{C}}>0.2 \epsilon_{\mathrm{C}_{\odot}}$. On the other hand; from (ii) and (iii) we may conclude that $\epsilon_{\mathrm{C}}<3 \epsilon_{\mathrm{C}_{\odot}}$. The C IV $\lambda \lambda 5801,5812$ lines also indicate that a $3 \epsilon_{\mathrm{C}_{\odot}}$ is an upper limit to the carbon abundance.

Our analysis indicates that the $08-9 \mathrm{~V}$ stars in our sample have a photospheric carbon abundance in the range $0.5 \lessgtr$ $\epsilon_{\mathrm{C}} / \epsilon_{\mathrm{C}_{\odot}} \lesssim 2$. Although the uncertainty is large, it is insufficient to allow the adoption of mass-loss rates that are consistent with the values predicted by theory $\left(\dot{M}_{\text {Vink }}\right)$. A simple test shows that even if a very low abundance of $0.2 \epsilon_{\mathrm{C}}$ is used along with $\dot{M}_{\text {Vink }}$, the model still shows a very intense C IV $\lambda \lambda 1548,1551$ P-Cygni profile which is not observed in any of the O8-9V stars. Despite the remaining uncertainty in the carbon abundance, which adds to the uncertainty in $\dot{M}$, a low carbon abundance cannot be invoked to solve the weak wind problem. A similar conclusion was also reached by Martins et al. (2005a) who quantified the error on the mass-loss rate, due to the uncertainties in the CNO abundances, to be about 0.3 dex.

\section{Mass-loss rates in late-type 0 dwarfs}

In most cases, the C IV $\lambda \lambda 1548,1551$ resonance doublet is the only diagnostic available to determine mass-loss rates for 

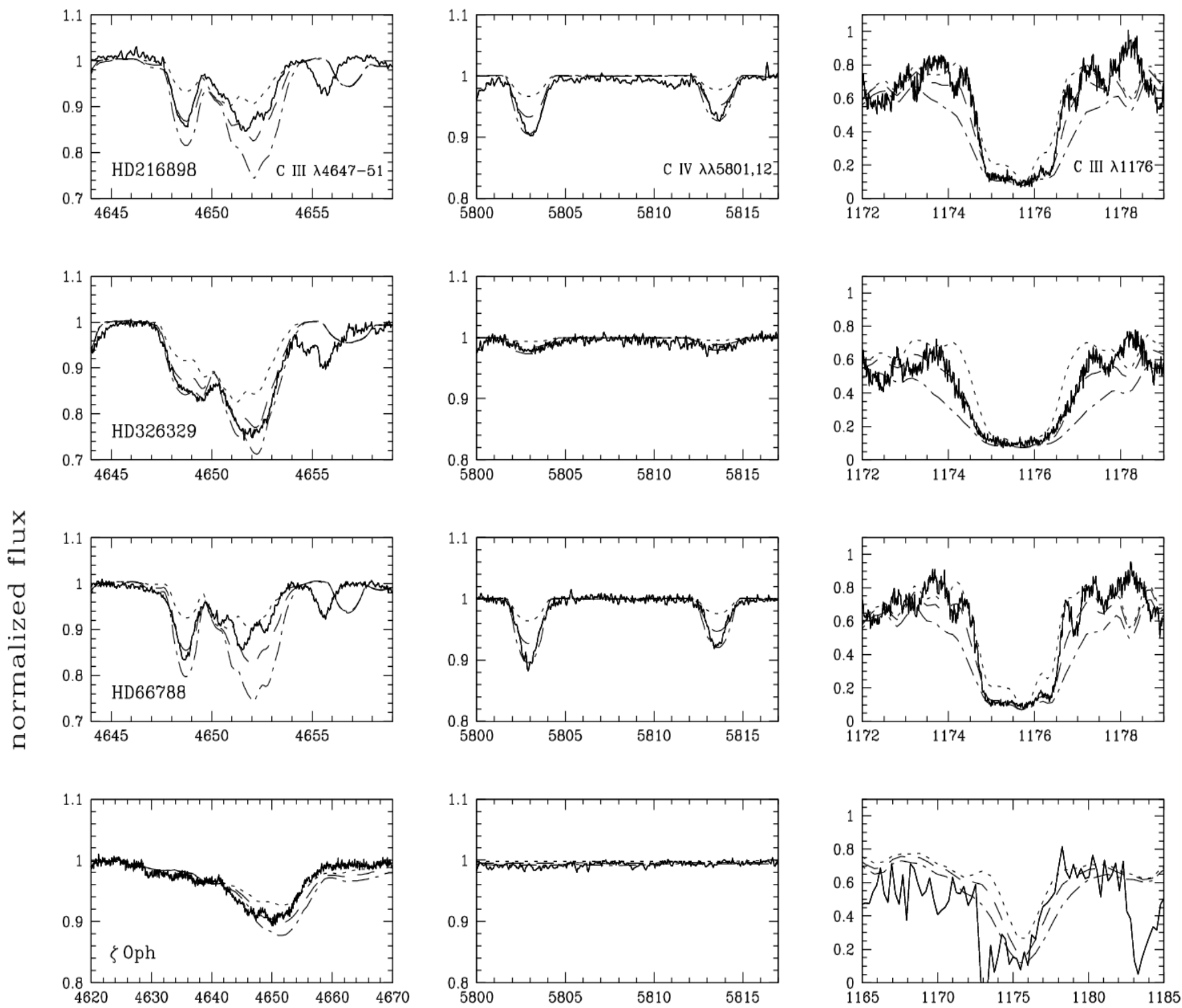

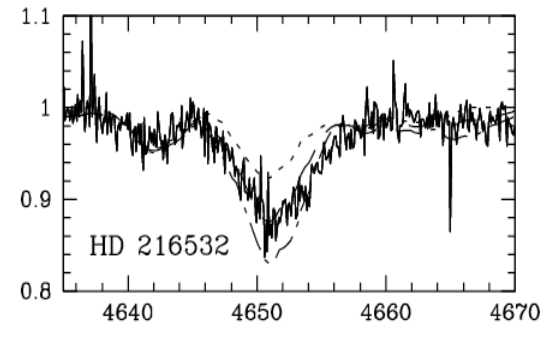

$\lambda(\AA)$
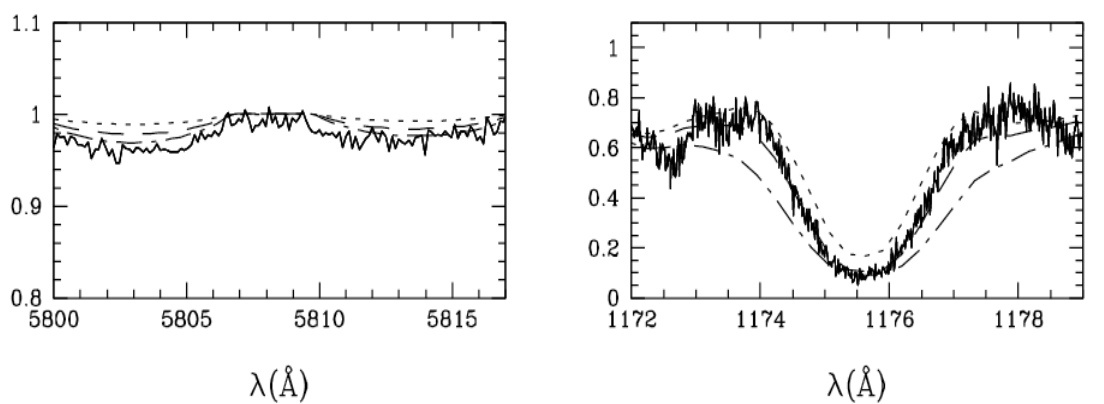

Fig. 2. Determination of the carbon abundance in our program stars. The observed spectra are indicated by solid lines. The models shown have: $20 \%$ solar $\left(0.2 \epsilon_{\mathrm{C}_{\odot}}\right.$; dotted line $)$, solar $\left(\epsilon_{\mathrm{C}_{\odot}} ;\right.$ dashed line $)$, and $3 \mathrm{x}$ the solar carbon abundance $\left(3 \epsilon_{\mathrm{C}_{\odot}} ;\right.$ dashed-dotted line $)$.

late-type $08-9 \mathrm{~V}$ stars. In order to overcome this limitation we have explored an alternative approach to estimate $\dot{M}$ values.

\subsection{Other $M$ diagnostics}

To provide additional mass-loss constraints we have proceeded in the following manner. For each object of our sample, we have started with the $\dot{M}$ determined from the best fit to
C IV $\lambda \lambda 1548,1551$ (the final model). We then gradually increased the mass-loss rate until reaching the value predicted by the radiative wind theory $\left(\dot{M}_{\text {Vink }}\right)$, which for each star is a function of $T_{\text {eff }}$, mass and luminosity. As soon as we used values higher than in our final models (yet considerably lower than $\dot{M}_{\text {Vink }}$ ), different lines started to reveal wind profiles that are not observed. We thus used this finding, and a variety of transitions, to establish upper limits on $\dot{M}$. 


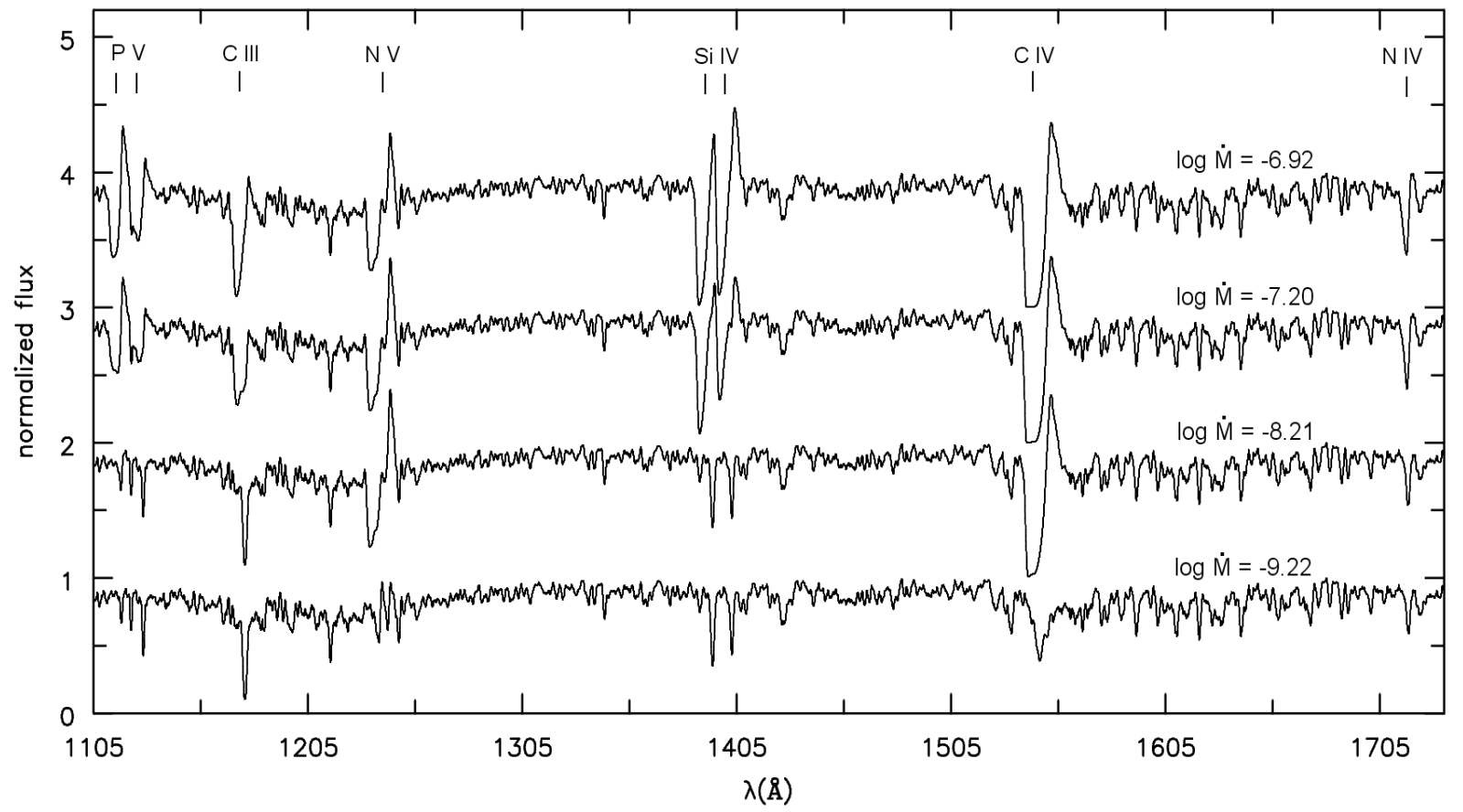

Fig. 3. $\dot{M}$-sensitive lines in the far-UV and UV regions. A $v \sin i$ convolution of $200 \mathrm{~km} \mathrm{~s}^{-1}$ was applied to each spectrum. Other physical parameters (e.g., $T_{\text {eff }}$ and $v_{\infty}$ ) are held fixed at the values derived for HD 216532 (see Table 3). The models were normalized and vertically displaced for clarity.

Table 4. Mass-loss rates and upper limits derived from different transitions (log units).

\begin{tabular}{lccccccc}
\hline \hline Star & $\dot{M}(\mathrm{C}$ IV $)$ & $\dot{M}(\mathrm{C}$ III $)$ & $\dot{M}(\mathrm{~N} \mathrm{~V})$ & $\dot{M}(\mathrm{~N} \mathrm{IV})$ & $\dot{M}(\mathrm{P} \mathrm{v})$ & $\dot{M}(\mathrm{Si}$ IV $)$ & $\dot{M}($ Vink $)$ \\
\hline HD 216898 & -9.35 & $<-7.47$ & $<-8.46$ & $<-7.80$ & $<-7.96$ & $<-7.59$ & -7.22 \\
HD 326329 & -9.22 & $<-7.73$ & $<-8.22$ & $<-7.49$ & $<-7.73$ & $<-7.96$ & -7.38 \\
HD 66788 & -8.92 & $<-7.22$ & $<-8.38$ & $<-7.52$ & $<-7.52$ & $<-7.22$ & -6.95 \\
J Oph & -8.80 & $<-7.40$ & $<-8.15$ & $<-6.89$ & $<-7.40$ & $<-7.70$ & -6.89 \\
HD 216532 & -9.22 & $<-7.45$ & $<-8.21$ & $<-7.57$ & $<-7.45$ & $<-7.70$ & -6.92 \\
\hline
\end{tabular}

In Fig. 3, we illustrate the $\dot{M}$ diagnostics found by following the aforementioned procedure. Besides C IV $\lambda \lambda 1548,1551$, the lines that show significant changes are: $\mathrm{P} V \mathrm{~V} \lambda 1118,1128$, C III $\lambda 1176, \mathrm{~N} v \lambda \lambda 1239,1243$, Si IV $\lambda \lambda 1394,1403$, and $\mathrm{N}$ IV $\lambda 1718$. When $\dot{M}$ is low (i.e., $\sim 10^{-10}-10^{-9} M_{\odot} \mathrm{yr}^{-1}$ ), $\mathrm{N}$ v $\lambda \lambda 1239,1243$ is generally weak (or absent) and the other lines are essentially of photospheric origin. However, as $\dot{M}$ is increased they show increasingly modified profiles. When $\dot{M}_{\text {Vink }}$ is used some lines show a very intense P-Cygni profile (for instance Si IV $\lambda \lambda 1394,1403)$.

The upper mass-loss rate limits $\left(\dot{M}_{\text {upper }}\right)$ for the lines were obtained in different ways. For P V $\lambda \lambda 1118,1128$, C III $\lambda 1176$, and Si IV $\lambda \lambda 1394,1403$, we determined when the profiles started to get filled by wind emission and/or when a blue absorption started to appear, deviating from the purely photospheric profiles that are observed. For N v $\lambda \lambda 1239,1243$, we used the strength of the whole P-Cygni profile, which generally is weak or absent in the observations. The N IV $\lambda 1718$ line has a different response. Instead of becoming filled in at its center, an asymmetric blueward absorption profile is formed. We then determined $\dot{M}_{\text {upper }}$ when a significant (blue) displacement from the observed feature was reached. Such behavior indicates that this line is formed in the very inner parts of the stellar wind. Hillier et al. (2003) found a similar situation in the homogeneous model for the $\mathrm{O} 7 \mathrm{Iaf}+$ star AV 83, especially S v $\lambda 1502$ (see their Fig. 10).
The results obtained for each object of our sample are summarized in Table 4. The C IV $\lambda \lambda 1548,1551$ mass-loss rates are those used for our best fit models presented in Sect. 4. The theoretical mass-loss rates are also shown for comparison. In Appendix B we present the models used to construct Table 4 and comment on each object.

In Fig. 4 we display the results graphically. The mass-loss rates are shown relative to the predicted value for each star. In all cases the upper limits on $\dot{M}$ fall below the theoretical line. The only exception is N IV $\lambda 1718$ for $\zeta$ Oph, for which we have assigned a conservative value. If we consider only the C IV $\lambda \lambda 1548,1551$ and N v $\lambda \lambda 1239,1243$ lines, most mass-loss rates are below -1.0 dex $\dot{M}_{\text {Vink }}$. Regarding the other transitions, the upper limits fall approximately at $(-0.5 \pm 0.2) \mathrm{dex}$, which implies that the mass-loss rates are still very low: they must be lower than $\dot{M}_{\text {Vink }}$ by at least a factor of two to five. Taken together these results provide, for the first time, additional support for the reality of weak winds.

If we focus only on one object at a time, it is also interesting to note from Fig. 4 that the points below the maximum value (i.e., closest to Vink's line) already have their theoretical line profiles in disagreement with the observed lines. In this sense, whenever we move to models/points higher than others, Fig. 4 can be interpreted as errors being accumulated in the model fits. 


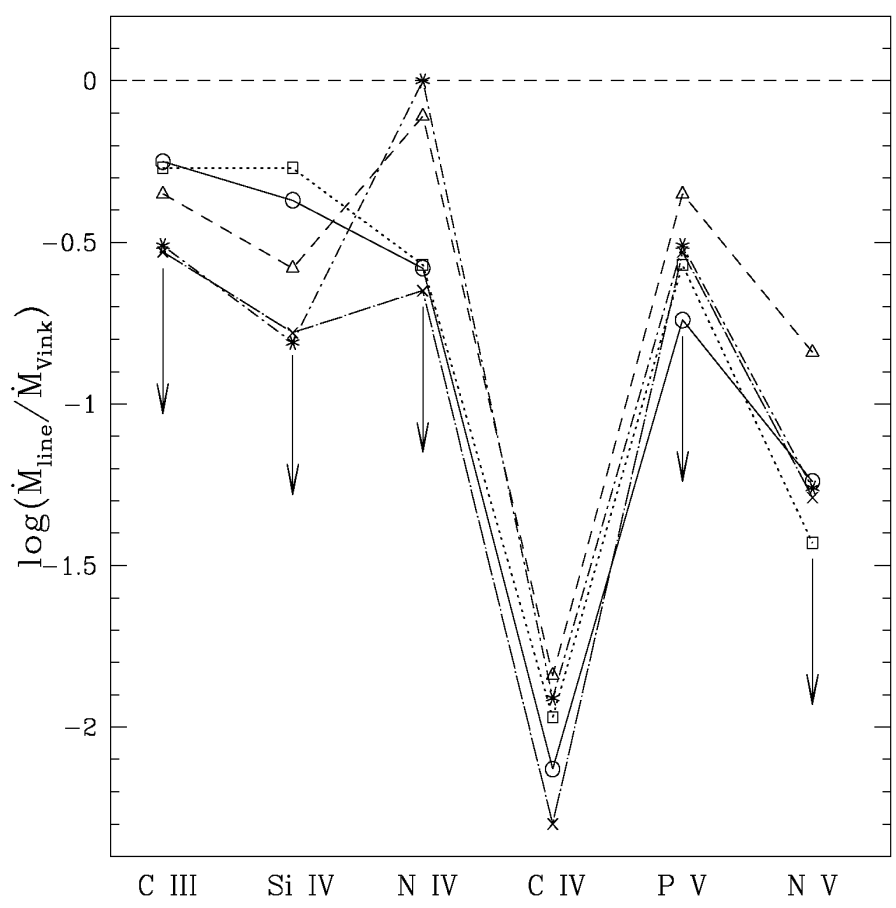

Fig. 4. Mass-loss rate upper limits derived from different transitions in the ultraviolet. The stars are: HD 216898 (circled+solid line), HD 326329 (triangles+dashed line), HD 66788 (squares+dotted line), $\zeta$ Oph (asterisks+dashed-dotted line), and HD 216532 (crosses+shortlong dashed line). Arrows indicate that the points represent upper limits.

We remind the reader that all the models presented in this paper do not include clumping, and thus some some of the upper limits presented above could actually be much lower. If the winds of the objects studied are not homogeneous we have:

$\log \frac{\dot{M}_{\text {clumped }}}{\dot{M}_{\text {Vink }}}=\log \frac{\dot{M}}{\dot{M}_{\text {Vink }}}+\log \sqrt{f}$,

where the first term on the right-hand side is in the vertical axis shown in Fig. 4. With a typical filling factor $f=0.1$, some points (depending on the wind clumping sensitivity of the respective lines) could go down, and hence away from Vink's prediction, by 0.5 dex. However, we think that it is more reasonable to compare the results of homogeneous atmosphere models with radiative wind models that are also homogeneous, as is the case in Vink et al. (2000, 2001).

\subsection{Ho behavior}

Although we determined mass-loss rates from the far-UV and UV spectral regions, we have also analyzed the sensitivity of $\mathrm{H} \alpha$ to changes in $\dot{M}$. This is important because, as highlighted in Sect. 5, the analysis of $\mathrm{H} \alpha$ by Mokiem et al. (2005) seems to indicate that a few stars do not have weak winds; the $\dot{M}$ values obtained are compatible with $\dot{M}_{\text {Vink }}$ (see also the discussion in Mokiem et al. 2007).

Previously, Martins et al. (2005a) already demonstrated two points regarding $\mathrm{H} \alpha$ : (i) in a low density wind $(\dot{M} \sim$ $10^{-9} M_{\odot} \mathrm{yr}^{-1}$ ) the CMFGEN and FASTWIND predictions agree; (ii) for $\dot{M}$ ranging from $\sim 10^{-10}$ to $10^{-9} M_{\odot} \mathrm{yr}^{-1}$ the resulting CMFGEN $\mathrm{H} \alpha$ profiles are virtually identical. Here, we extend their analysis by presenting the changes in the $\mathrm{H} \alpha$ profile for a wider range in mass-loss rates: from $\sim 10^{-10}$ to $10^{-6} M_{\odot} \mathrm{yr}^{-1}$.

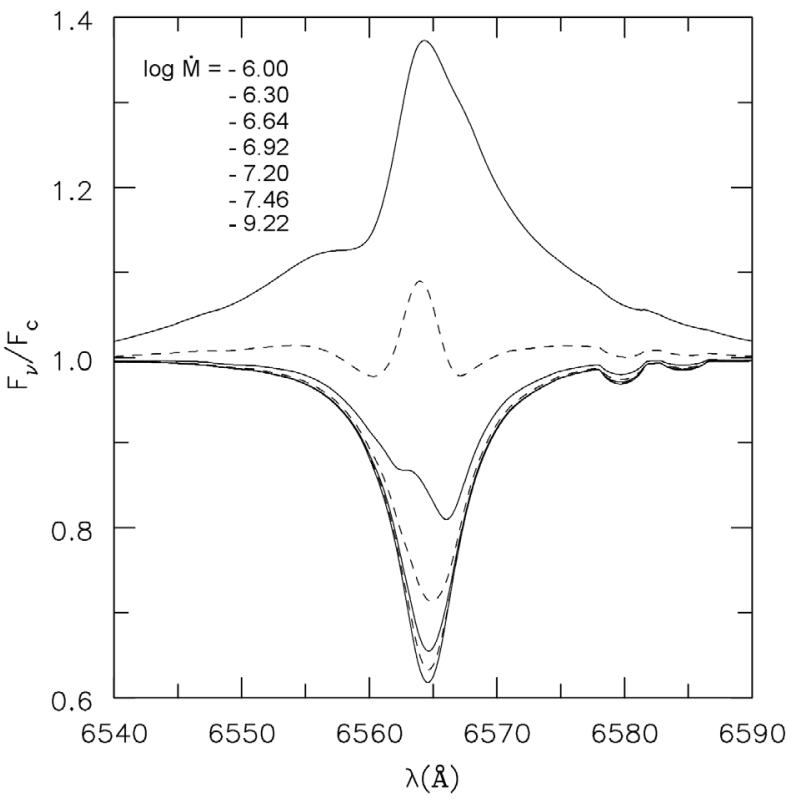

Fig. 5. Sensitivity of $\mathrm{H} \alpha$ to different values of the mass-loss rate. The model with the lowest mass-loss rate has $\log \dot{M}=-9.22$ and corresponds to the most intense absorption line. The model with the highest mass-loss rate has $\log \dot{M}=-6.0$; it corresponds to the most intense line in emission. For clarity, intermediate mass-loss rate models are shown by alternating dashed and full lines.

In Fig. 5 we display models with the photospheric parameters fixed ${ }^{5}$ and different values for the mass-loss rate. Only for $\log \dot{M} \gtrsim-6.30$ is $\mathrm{H} \alpha$ in emission. For lower rates, the predicted profiles are in absorption and therefore the measurement of $\dot{M}$ must be based on the filling of the photospheric profile. In the example shown, models with a $\log \dot{M}=-9.22, \log \dot{M}=-7.46$, and $\log \dot{M}=-7.20$ do not differ much. Despite a factor of one hundred change in $\dot{M}$, the changes in the profiles are too small to allow a secure discrimination of a best fit model to an observed spectrum. Overall, the models suggest that we can only have confidence in $\mathrm{H} \alpha$ based mass-loss rates when $\dot{M} \gtrsim 10^{-7} M_{\odot} \mathrm{yr}^{-1}$. As can be seen from Fig. 5, noticeable changes start to be found only above this threshold.

The fits of $\mathrm{H} \alpha$ using our final models and models with $\dot{M}_{\text {Vink }}$ are shown in Fig. 6 (see Table 3 for the corresponding values). Focusing first on HD 216898 and HD 66788, neither our final models nor the ones using $\dot{M}_{\text {Vink }}$ match the observed $\mathrm{H} \alpha$. The models using $\dot{M}_{\text {Vink }}$ are weaker while our final models are stronger than the observed line center. However, the differences compared to the observations are very small: $\sim 0.02$ of the continuum intensity. Given the uncertainties involved in the normalization of the spectra of our sample around $\mathrm{H} \alpha$, we find it very hard to choose with certainty between any of these models. As in Martins et al. (2005a), we estimate that the position of the line core can have an error up to $2 \%$. However, if a nebular contamination is likely or the signal-to-noise ratio of the spectra is not high enough, the uncertainty increases considerably. Such situations illustrate why it is not straightforward to establish $\dot{M}$ values for O8-9V stars using $\mathrm{H} \alpha$. For HD 326329 the situation is similar, but the $\dot{M}_{\text {Vink }}$ model presents a better fit than our final model.

Interestingly, for $\zeta$ Oph and HD 216532, our final models seem to be better than the ones using $\dot{M}_{\text {Vink }}$, and not only in the line center. With the predicted mass-loss rates for these stars we

\footnotetext{
${ }_{5}^{5}$ In this example $v \sin i=80 \mathrm{~km} \mathrm{~s}^{-1}$ and the others parameters are fixed at the values derived for HD 216532.
} 

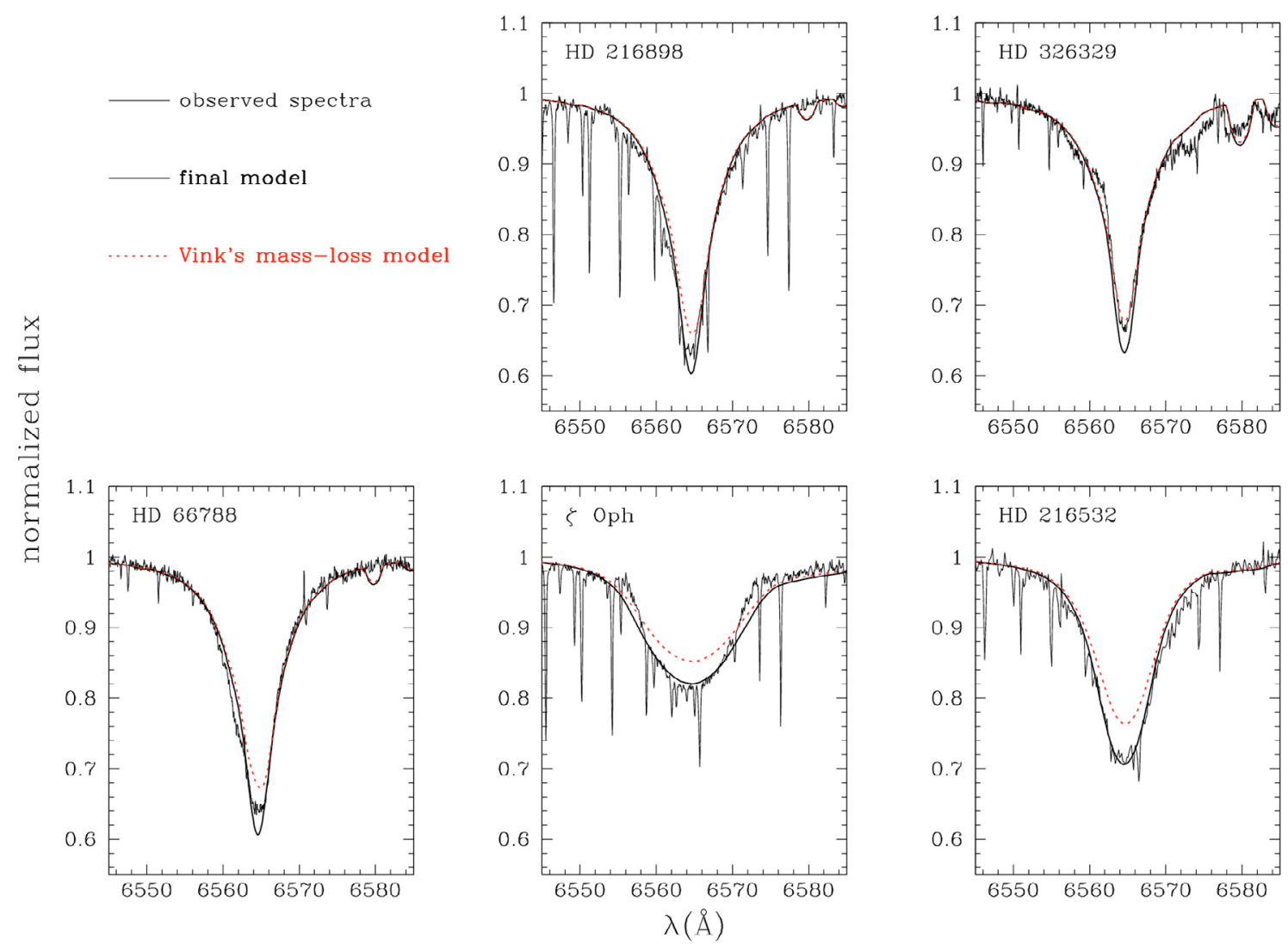

Fig. 6. Fits to the observed $\mathrm{H} \alpha$ lines in our sample using the mass-loss rates of our final models and the mass-loss rates predicted by theory $\left(\dot{M}_{\text {Vink }}\right)$.

cannot fit either the UV or the optical lines. Repolust et al. (2004) and Mokiem et al. (2005) derived $\dot{M}$ for $\zeta$ Oph based on $\mathrm{H} \alpha$ fits with the FASTWIND code. The former authors derived an upper limit of $\log \dot{M}=-6.74$ and the latter found a mass-loss rate of $\log \dot{M}=-6.85$. In both studies, the velocity law $\beta$ parameter was fixed at 0.8. While the fit in Fig. 6 uses $\beta=1$, we also tested a model with the same predicted mass- $\operatorname{loss}\left(\log \dot{M}_{\mathrm{Vink}}=-6.89\right)$ and $\beta=0.8$. The synthetic $\mathrm{H} \alpha$ line shows a deeper core, but it is still not deep enough to fit the observations. We therefore cannot support the findings of Mokiem et al. (2005). We speculate that one reason for this discrepancy may be the use of different data. An alternative reason could be the existence of a discrepancy between the predicted $\mathrm{H} \alpha$ profile by CMFGEN and FASTWIND at $\sim 10^{-7} M_{\odot} \mathrm{yr}^{-1}$. In a comparison presented by Puls et al. (2005) it was shown that these two codes can predict slightly different $\mathrm{H} \alpha$ line intensities. In the case of an O8V or an O10V model for instance (see their Fig. 17), FASTWIND tends to predict a stronger $\mathrm{H} \alpha$ absorption than CMFGEN. Such differences will affect mass-loss rate measurements for late $\mathrm{O}$ dwarfs obtained from the $\mathrm{H} \alpha$ absorption line.

\section{Discussion}

A large discrepancy is found between the models and the observations (see Figs. B.1-B.5) when mass-loss rates higher than those derived from C IV $\lambda \lambda 1548,1551$ are used. Most importantly, this mismatch is found for lines of different ions, and for every object in our sample. With these different diagnostics, the existence of weak winds gains strong additional support.

What are the consequences if we accept the mass-loss rates predicted by Vink et al. (2000), for late O dwarfs, as correct? Given our findings, we would have to conclude that the expanding atmosphere models we used lack essential physics or are based on incorrect assumptions or approximations (or both). The model wind structure/ionization must be altered in a drastic way for the model with the theoretical mass-loss rate to predict only a weak C IV $\lambda \lambda 1548,1551$ wind feature (and perhaps also a weak $\mathrm{N} v \lambda \lambda 1239,1243)$. An example of what is needed spectroscopically is illustrated by Fig. 3. The uppermost synthetic spectrum shown in Fig. 3 would have to be transformed into an essentially photospheric spectrum (lower spectrum). Although possible, this scenario presents several difficulties.

First and foremost, contrary to the recently reported case of some B supergiants where a few key UV wind lines could not be reproduced by CMFGEN models (see Searle et al. 2008; and also Crowther et al. 2006) ${ }^{6}$, we find agreement with the observations (see Figs. A.1-A.10). Although in most spectra only a single conspicuous wind signature is observed, the lack of wind lines such as P V $\lambda \lambda 1118,1128$, C III $\lambda 1176$, Si IV $\lambda \lambda 1394,1403$, and N IV $\lambda 1718$, also constrains our models. These transitions are mostly from the photosphere (which is smoothly connected to the wind), and they can be well reproduced.

In addition, the reasonable fits that we achieved with very low mass-loss rates would have to be merely an unfortunate coincidence. This would also be true for the stars analyzed by Martins et al. (2005a). Furthermore, if there were an issue with some missing physical ingredient(s), or with some assumptions that need to be revised, the theoretical hydrodynamical predictions would also need to be reassessed. We could then neither

${ }^{6}$ For B stars a problem with the model wind ionization structure has been put forward, although the wind structure of B supergiants differs from the wind in O stars (Prinja et al. 2005). X-rays, however, were not used in their analyses, and their inclusion might help to resolve some of the discrepancies. 


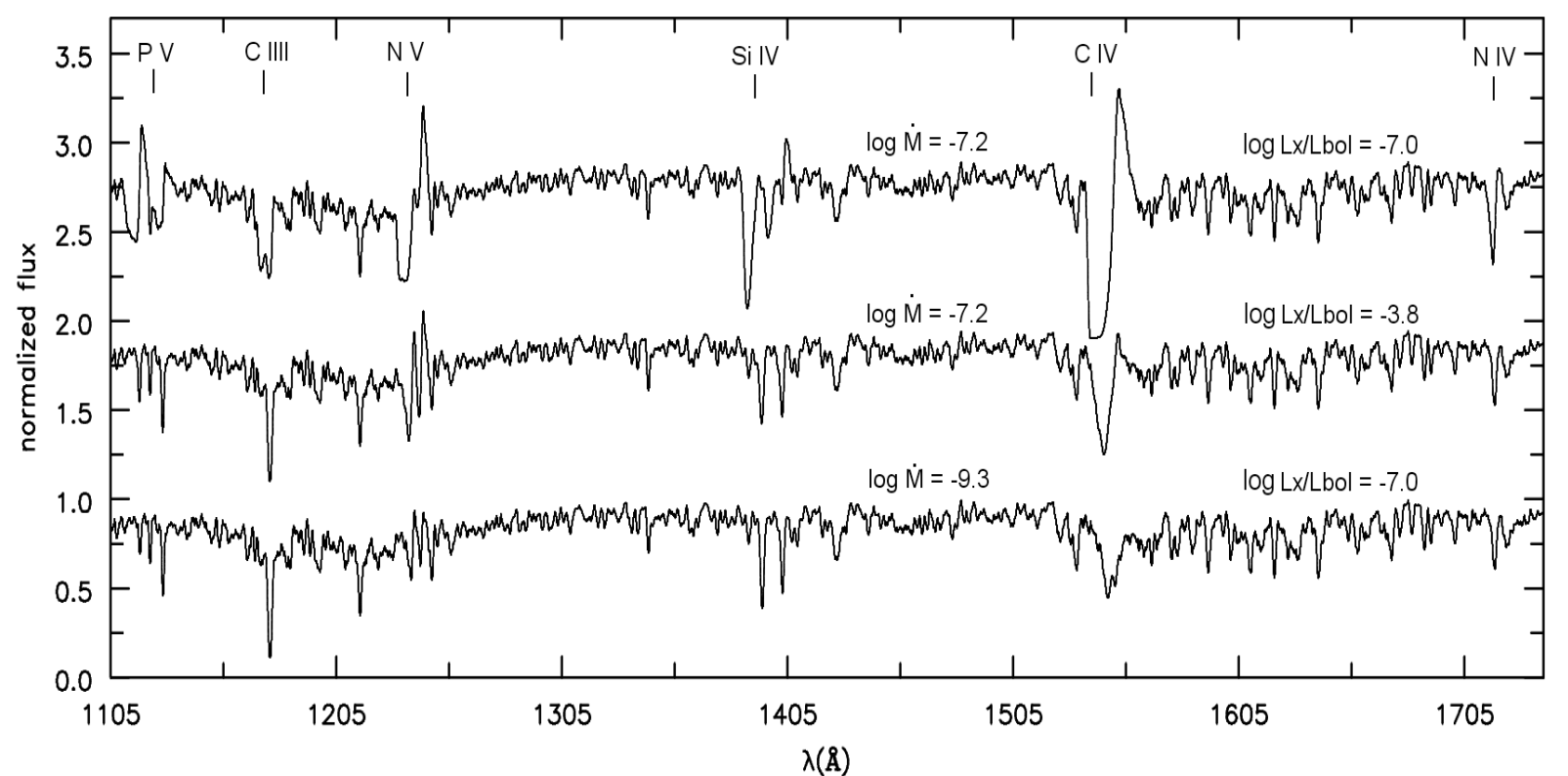

Fig. 7. Effects of different $\log L_{\mathrm{X}} / L_{\mathrm{Bol}}$ ratios in the ultraviolet synthetic spectrum. Note the similarities between the middle and bottom spectra, despite the very different mass-loss rates.

trust the results from the atmosphere models nor the current radiative wind models when considering late $\mathrm{O}$ dwarfs. The weak wind problem would become ill-defined. Future, more sophisticated models that could achieve fits of similar quality to our fits would most likely require additional free input parameters or assumptions (e.g., non-spherical winds).

There is no clear answer as to the missing ingredient(s) in the current atmosphere models. Although CMFGEN and other codes such as FASTWIND are considered state-of-the-art for hot massive stars, several physical phenomena are treated only approximately, or are not taken into account, due to technical challenges and a lack of observational constraints (e.g., nonstationarity; wind rotation; non-sphericity; realistic description of clumping; see Hillier 2008, and references therein). It is possible that advances in some of these areas will cast new light on the analysis of massive stars. However, the consequences regarding the weak wind problem are presently difficult to assess.

In short, we believe that the inclusion of new physics or the relaxation of standard assumptions deserves to be addressed in future studies (with the appropriate observational constraints), but our model atmospheres for the O8-9V stars are the best currently available.

Below we speculate about some possibilities worthy of investigation, and thereafter discuss the hypothesis that the low density winds are highly ionized due to X-ray ionization.

\subsection{Magnetic fields and multi-component winds}

From the best-fit models, we can examine how closely the momentum absorbed by the wind matches that required to satisfy the momentum equation. In general, for the 08-9V stars, we find that the wind absorbs too much momentum. This can be illustrated by noting that the mass-loss rate from a single saturated line is approximately $L / c^{2}$ (Lucy \& Solomon 1970). In all cases, this value exceeds our best fit mass-loss rates (see Table 3). While none of the wind lines in the models are saturated, there are sufficient weak lines to yield a force similar, or larger, than the single line limit.
The cause of the discrepancy is unclear. Possibilities include the existence of magnetic fields, and/or the presence of a significant component of hot gas. The latter could be generated by shocks in the stellar wind. As noted by Drew et al. (1994) and Martins et al. (2005a), it is possible that at the densities encountered in these winds the shocked gas never cools (see also Krtička \& Kubát 2009). Such gas would not be easily detectable in the UV since it would be collisionally ionized to such an extent that very few C IV and N V ions would exist. Further evidence for a significant amount of hot gas comes from the observed X-ray fluxes; the required filling factors in the models (while strongly mass-loss rate dependent) indicate that the hot gas in the wind is not a trace component.

Of interest in this regard are the distinct profiles presented by C IV $\lambda \lambda 1548,1551$. While all show blue-shifted absorptions, the depth and shapes differ significantly among the stars (see for instance the ones in HD 66788, HD 326329, and HD 216532). Also of concern is the general weakness of the P-Cygni emission components (with the possible exception of $\zeta$ Oph). Instead of very low mass-loss rates, such an absence might be related to complicated wind structures.

\subsection{Winds highly ionized by $X$-rays}

Although good fits could be achieved with very low $\dot{M}$ 's, we also investigated alternative models that might give agreement with the observations using $\dot{M}_{\text {Vink }}$. It soon became clear that a promising approach was to explore high levels of X-ray emission. If most of the wind is highly ionized, we do not expect significant wind emission in the UV lines.

In order to test this idea, we explored models with high values for the $L_{\mathrm{X}} / L_{\mathrm{Bol}}$ ratio. We found that the wind emission is decreased in the desired manner. To illustrate this we present, in Fig. 7, two models with the same mass-loss rate (typically $\dot{M}_{\text {Vink }}$ ) but with different values of $\log L_{\mathrm{X}} / L_{\mathrm{Bol}}$ : the canonical value of -7.0 and a much higher ratio of -3.8 . In addition, a third model with a very low mass-loss rate $(\log \dot{M}=-9.3$; as in a typical final model) is shown with $\log L_{\mathrm{X}} / L_{\mathrm{Bol}}=-7.0$. Because of the high level of $\mathrm{X}$-ray emission, the model with 
$\log L_{\mathrm{X}} / L_{\mathrm{Bol}}=-3.8$ has most of its wind highly-ionized and, despite the high mass-loss rate, no significant UV line emissions are predicted. Indeed, the synthetic spectrum with high $\dot{M}$ and high $\log L_{\mathrm{X}} / L_{\mathrm{Bol}}$ resembles the one with very low $\dot{M}$ and normal $\log L_{X} / L_{\text {Bol }}$ (see the spectra in the middle and bottom of Fig. 7).

In order to better quantify the result above, we have turned our attention to the ionization fractions of $\mathrm{P} \mathrm{V}, \mathrm{N} \mathrm{V}$, Si IV, and C IV. For the resonance lines P V $\lambda \lambda 1118,1128$, N v $\lambda \lambda 1239,1243$, Si IV $\lambda \lambda 1394,1403$, and C IV $\lambda \lambda 1548,1551$, it is well known that the crucial parameter needed to fit the profiles is the line optical depth which is directly proportional to the mass-loss rate and the respective ionization fraction (see for instance the case of $\mathrm{P} V \lambda \lambda 1118,1128$ in Fullerton et al. 2006).

Thus, our final models actually provide ${ }^{7}$

$\dot{M} \times q_{i}=\Psi_{i}$

where $\Psi_{i}$ is a constant that depends on the observed spectrum and $q_{i}$ is the ionization fraction of the ion $i$. This latter is usually defined as:

$q_{i}=\frac{\int_{0.2}^{1} n_{i}(x) \mathrm{d} x}{\int_{0.2}^{1} n(x) \mathrm{d} x}$,

where $x=v(r) / v_{\infty}$ and $n_{i}$ and $n$ are the ion and element number density, respectively. We can now compute the ionization fraction needed to fit the observations by using $\dot{M}_{\text {Vink }}$, which we call $q_{i, \text { Vink }}$. Since the constant $\Psi_{i}$ gives the appropriate fit to the observations, we can write:

$q_{i, \mathrm{Vink}}=\Psi_{i} / \dot{M}_{\mathrm{Vink}}$.

After deriving $q_{i \text {, Vink }}$, for each ion from the above equation, we computed several models with the mass-loss rate fixed at $\dot{M}_{\text {Vink }}$ but with different values of $\log L_{\mathrm{X}} / L_{\mathrm{Bol}}$. For each model, we then derived the ionization fraction of each ion following Eq. (2). We call these ionization fractions $q_{\mathrm{X}}$ (i.e., the ionization fractions for a specific $\log L_{\mathrm{X}} / L_{\mathrm{Bol}}$ value). We determined when $q_{\mathrm{X}}$ was equal to $q_{i \text {,Vink }}$, in order to find the level of X-ray emission needed to obtain agreement with the observations using $\dot{M}_{\text {Vink }}$.

In Fig. 8 we plot the ratio $q_{i, \text { Vink }} / q_{\mathrm{X}}$ for each ion versus several $\log L_{\mathrm{X}} / L_{\mathrm{Bol}}$ values. For simplicity, we discuss only one object in our sample, HD 216898. Figure 8 shows that all ions do not behave exactly in the same manner. This is not surprising, since they have different electronic structures and ionization potentials, and thus react differently to $\mathrm{X}$-rays. If we focus on $\mathrm{C}$ IV and $\mathrm{P} \mathrm{V}$, we see that only very high $\log L_{\mathrm{X}} / L_{\mathrm{Bol}}$ ratios (around -3.0 ) give $q_{\mathrm{X}}$ close to $q_{i \text {,Vink }}$. Otherwise the ratio, $q_{i, \text { Vink }} / q_{\mathrm{X}}$, is much lower than unity. In the case of $\mathrm{Si}$ IV, $q_{i, \mathrm{Vink}} / q_{\mathrm{X}}$ remains very small regardless of the value of $\log L_{\mathrm{X}} / L_{\mathrm{Bol}}$. An increase, however, is found for $\log L_{\mathrm{X}} / L_{\mathrm{Bol}} \approx-3.0$. For $\log L_{\mathrm{X}} / L_{\mathrm{Bol}} \sim-7$, we have $q_{i, \text { Vink }} / q_{\mathrm{X}} \sim$ $5 \times 10^{-5}$, but for $\log L_{\mathrm{X}} / L_{\mathrm{Bol}} \sim-3$ this ratio is much higher, $q_{i, \text { Vink }} / q_{\mathrm{X}} \sim 0.005$. We therefore conclude for this ion that even more X-rays seem to be required for $q_{\mathrm{X}}$ to reach $q_{i \text {,Vink }}$. The situation for $\mathrm{N} v$ is quite interesting: for both low and high values of $\log L_{\mathrm{X}} / L_{\mathrm{Bol}}$, the $q_{\mathrm{X}}$ approaches $q_{i \text {,Vink }}$. This, however, is not hard to explain. For high values of $\log L_{X} / L_{\mathrm{Bol}}$ practically all the nitrogen is concentrated in N VI. The ionization fraction $q_{\mathrm{X}}$ obtained for $\mathrm{N} \mathrm{V}$ is as low as the ionization fraction of Vink, $q_{\text {Vink }}$, computed from Eq. (3). On the other hand, when very low

\footnotetext{
7 If the ionization fractions are reliable we get $\dot{M}$ from the model fits, otherwise we get $\Psi_{i}$.
}

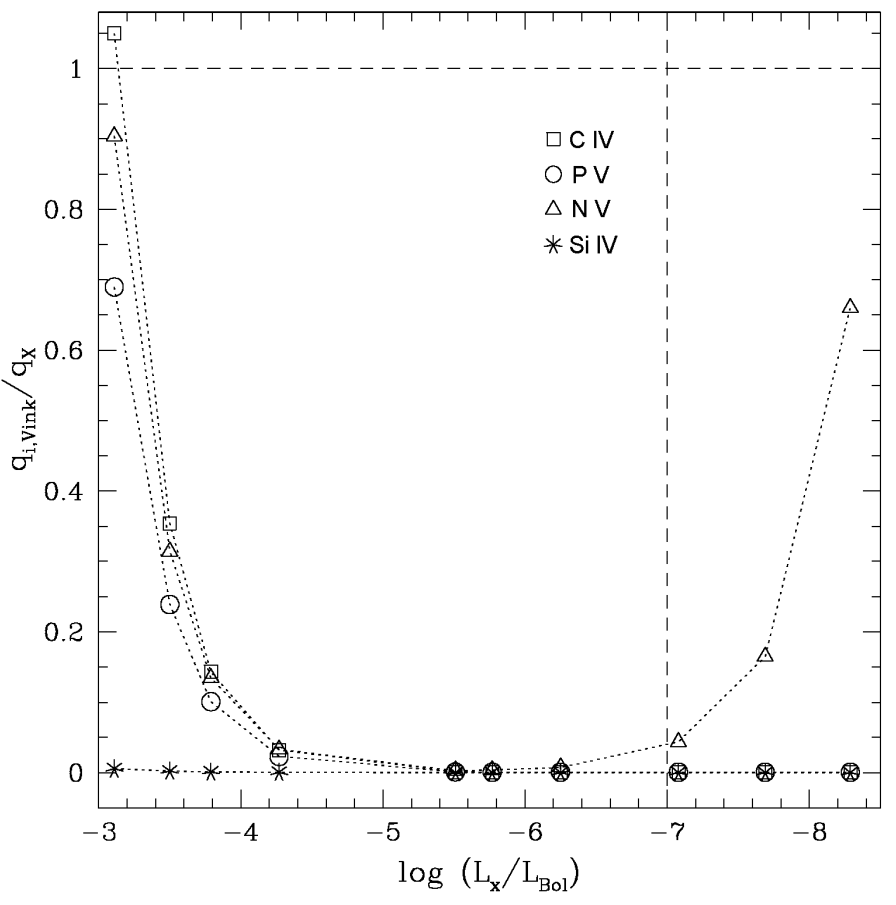

Fig. 8. Ionization fractions as a function of $\log L_{\mathrm{X}} / L_{\mathrm{Bol}}$. The values for $q_{\mathrm{X}}$ were computed using Eq. (2) and $\dot{M}_{\text {Vink }}$. The $q_{i, \text { Vink }}$ values were derived from Eq. (3) (see text for more details).

$\log L_{\mathrm{X}} / L_{\mathrm{Bol}}$ ratios are considered, we verified that most of the nitrogen is in the $\mathrm{N}$ III-IV states. Thus, again, a very low $q_{\mathrm{X}}$ is obtained for $\mathrm{N} \mathrm{V}$. In practice, this means that the observed, weak N v $\lambda \lambda 1239,1243$ line (or its absence), can be reproduced by models using $\dot{M}_{\text {Vink }}$ with either very low or large amounts of $\mathrm{X}$-rays.

We conclude from both Figs. 7 and 8 that the only way to match the observed spectra with high mass-loss rate $\left(\dot{M}_{\text {Vink }}\right)$ models is to adopt $\log L_{\mathrm{X}} / L_{\mathrm{Bol}} \gtrsim-3.5$. Such high values are however not supported by X-ray observations of O stars, where the measured values are typically around $\log L_{\mathrm{X}} / L_{\mathrm{Bol}} \approx-7.0$ (see e.g., Sana et al. 2006) with a scatter of about \pm 1.0 dex. Because of the importance for the wind ionization structure, future studies of different scenarios for the emission of X-rays in stellar winds are warranted.

\section{Conclusions}

We analyzed a sample of five late-type Galactic O dwarfs (HD 216898, HD 326329, HD 66788, $\zeta$ Oph, and HD 216532) using atmosphere models computed with the CMFGEN and TLUSTY codes. Model fits for the far-UV, UV and optical spectra were presented, and stellar and wind parameters were derived. The mass-loss rates were first derived using the C IV $\lambda \lambda 1548,1551$ doublet and we then explored other diagnostic lines. Our main motivation to study O8-9V stars was to address the so-called weak wind problem, recently introduced in the literature. The main findings of our study are summarized below:

$\triangleright$ The stellar parameters obtained for our sample are fairly homogeneous (see Table 3). Surface gravities of about 3.8 (af-

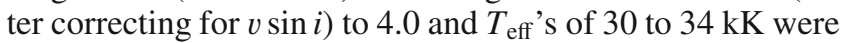
obtained. These values show good agreement with the latest calibrations of Galactic O star parameters for the spectral types of the program stars. 
$\triangleright$ Using the C IV $\lambda \lambda 1548,1551$ line we derived mass-loss rates considerably lower than the theoretical predictions of Vink et al. (2000). A discrepancy of roughly two orders of magnitude is found (see Fig. 1). We thus confirm the results of the study of Martins et al. (2005a) that Galactic O8-9 dwarf stars have weak winds. We also confirm a breakdown or a steepening of the modified wind momentum luminosity relation for low luminosity objects $\left(\log L_{\star} / L_{\odot} \lesssim 5.2\right)$.

$\triangleright$ We investigated the carbon abundance using a set of UV (C III 11176) and optical photospheric lines. We estimated that the following range is reasonable for our program stars: $0.5 \lesssim \epsilon_{\mathrm{C}} / \epsilon_{\mathrm{C}_{\odot}} \lesssim 2$ (in mass fractions). Although the uncertainty is large, it is not large enough to allow a solution of the weak wind problem.

$\checkmark$ Different methods to determine mass-loss rates for late $\mathrm{O}$ dwarfs were explored. We found that $\mathrm{P} \vee \lambda \lambda 1118,1128$, C III $\lambda 1176, \mathrm{~N}$ v $\lambda \lambda 1239,1243$, Si IV $\lambda \lambda 1394,1403$, and $\mathrm{N}$ IV $\lambda 1718$ lines are good diagnostic tools, and we used each of them to derive independent mass-loss rate limits for each star in our sample. We found that together with C IV $\lambda \lambda 1548,1551$, the use of the $\mathrm{N} v \lambda \lambda 1239,1243$ line gives the lowest $\dot{M}$ values: they must be less than about -1.0 dex $\dot{M}_{\text {Vink }}$. By considering the other lines, we still find very low mass-loss rates. They show that $\dot{M}$ must be lower than about $(-0.5 \pm 0.2)$ dex $\dot{M}_{\text {Vink }}$ (i.e., at least a factor of two to five lower than predicted). These results bring additional support for the reality of weak winds.

$\triangleright$ Upper mass-loss rate limits derived from N IV $\lambda 1718$ and C III $\lambda 1176$ can be a factor of three, or more, lower than the theoretical mass-loss rates of Vink. This is crucial in confirming a mass-loss discrepancy as $\mathrm{N}$ IV $\lambda 1718$, and to a lesser extent C III $\lambda 1176$, are formed close to the photosphere, where the uncertainties in the ionization structure should be much less than in the (outer) wind.

$\triangleright$ We analyzed the $\mathrm{H} \alpha$ line and found that its profile is insensitive to $\dot{M}$ when $\dot{M}<10^{-8} M_{\odot} \mathrm{yr}^{-1}$. For some objects of our sample we are unable to choose between the $\mathrm{H} \alpha$ fit presented by our final models and the ones obtained using $\dot{M}_{\text {Vink }}$. The interpretation of the results is further hindered by uncertainties in the continuum normalization of echelle spectra, and possible contamination of the core of $\mathrm{H} \alpha$ by nebular emission. For HD 216532 and $\zeta$ Oph the fits to the observed H $\alpha$ lines using $\dot{M}_{\text {Vink }}$ were not satisfactory. This result shows that even when $\mathrm{H} \alpha$ is used, lower than predicted mass-loss rates can be preferred.

$\triangleright$ We investigated ways for the model predictions to give agreement with the observed spectra of O8-9V stars using the mass-loss rates predicted by theory, and still assuming a "cold" wind. The only mechanism that we found is X-rays. By using high values for the $\log L_{\mathrm{X}} / L_{\mathrm{Bol}}$ ratio very little wind emission occurs, as in models with very low mass-loss rates $\left(\sim 10^{-10}-10^{-9} M_{\odot} \mathrm{yr}^{-1}\right)$. However, the values needed to be used ( $\left.\log L_{\mathrm{X}} / L_{\mathrm{Bol}} \gtrsim-3.5\right)$ are not supported by the observations, which usually measure $\log L_{\mathrm{X}} / L_{\mathrm{Bol}}$ values near -7.0 .

Although our analysis was performed with state-of-the-art atmosphere models, there are issues which still need to be addressed in future studies. For example, some stars of our sample (HD 326329 and HD 216532) show a deep C IV $\lambda \lambda 1548,1551$ absorption feature that could not be reproduced. The origin of this extra absorption remains to be investigated (see Sect. 8.1). Studies concerning non-spherical winds, especially for stars that are fast rotators, are also of great interest. Synthetic spectra computed by our group, using the 2D modeling approach of
Busche \& Hillier (2005), have shown interesting line-profile changes. A deeper analysis is needed to see if some of the discrepancies found in the case of $\zeta$ Oph can be solved. Furthermore, a more realistic treatment or even alternative descriptions of the X-ray emission in the atmosphere models (e.g., in conformity with the magnetic confinement scenario of Ud-Doula \& Owocki 2002; see also Waldron \& Cassinelli 2009) could bring valuable information. First steps in this direction have been taken by Zsargó et al. (2008). As we have shown in Sect. 8.2, X-rays are an efficient mechanism to change the ionization structure of the stellar winds.

It would also be very useful to analyze a sample of $\mathrm{O}$ stars having $\log L_{\star} / L_{\odot}$ near $\sim 5.2$. The disagreement between atmosphere models and theoretical predictions for Galactic stars seems to start around this value. The candidate spectral types would be $06.5 \mathrm{~V}, 07 \mathrm{~V}$, and $07.5 \mathrm{~V}$. Also, this same question needs to be better studied in metal-poor environments such as the LMC and SMC. We intend to investigate these issues in a future paper.

There are also important hydrodynamical questions that need to be addressed. In the work of Vink et al. (1999, 2000, 2001), the theoretical mass-loss rate is based on a set of ISA-WIND models (de Koter et al. 1997) and the use of a Monte-Carlo technique to compute the radiative acceleration. Although the results obtained by their procedure are self-consistent, the ISAWIND atmosphere models do not include the effect of X-rays. As the wind ionization can be changed significantly by X-rays, new radiative accelerations can be found and perhaps new predicted mass-loss rates might be derived. Within this picture, the agreement with the Vink et al. predictions for early $\mathrm{O}$ dwarfs may be understood, since their winds are not seriously affected by X-rays. Another hydrodynamical issue that needs to be addressed is the hot gas generated by the shocks. How efficiently does the hot gas cool, what role does the hot gas play in the wind dynamics, and can the hot gas still be considered as an impurity component of the stellar wind?

Our results bring new constraints to the weak wind problem. Galactic 08-9V stars seem to present very low mass-loss rates, as indicated by the C IV $\lambda \lambda 1548,1551$ doublet profile and other spectral diagnostics. The existence of weak winds poses a challenge to the current radiative wind models; this may have important consequences for our understanding of massive star evolution.

Acknowledgements. W.M. acknowledges the travel grant provided by IAU (Exchange of Astronomers Program) and CNES for the postdoctoral fellowship. J.-C.B. acknowledges financial support from the French National Research Agency (ANR) through program number ANR-06-BLAN-0105. T.L. and D.J.H. were supported by the NASA Astrophysics Data Program (grant NNG04GC81G). We wish to thank Yaël Nazé for helping with the normalization of the spectrum of HD 216898. We also wish to thank an anonymous referee for useful comments which helped to improve the paper. This research has made use of the SIMBAD database, operated at CDS, Strasbourg, France.

\section{References}

Abbott, D. C. 1982, ApJ, 263, 723

Baranne, A., Queloz, D., Mayor, M., et al. 1996, A\&AS, 119, 373 Baume, G., Vázquez, R. A., \& Feinstein, A. 1999, A\&AS, 137, 233

Bouret, J.-C., Lanz, T., Hillier, D. J., et al. 2003, ApJ, 595, 1182

Bouret, J.-C., Lanz, T., \& Hillier, D. J. 2005, A\&A, 438, 301

Busche, J. R., \& Hillier, D. J. 2005, AJ, 129, 454

Cardelli, J. A., Clayton, G. C., \& Mathis, J. S. 1988, ApJ, 329, L33

Cohen, D. H. 2008, Massive Stars as Cosmic Engines, Proceedings of the International Astronomical Union, IAU Symp., 250, 17

Crowther, P. A., Lennon, D. J., \& Walborn, N. R. 2006, A\&A, 446, 279 
de Koter, A., Heap, S. R., \& Hubeny, I. 1997, ApJ, 477, 792

Drew, J. E., Hoare, M. G., \& Denby, M. 1994, MNRAS, 266, 917

Ebbets, D. 1981, PASP, 93, 119

Escolano, C., et al. 2008, in prep.

Frémat, Y., Zorec, J., Hubert, A.-M., \& Floquet, M. 2005, A\&A, 440, 305

Freyer, T., Hensler, G., \& Yorke, H. W. 2003, ApJ, 594, 888

Fullerton, A. W., Massa, D. L., \& Prinja, R. K. 2006, ApJ, 637, 1025

García, B., \& Mermilliod, J. C. 2001, A\&A, 368, 122

Garmany, C. D., \& Stencel, R. E. 1992, A\&AS, 94, 211

Garrison, R. F. 1970, AJ, 75, 1001

Grevesse, N., \& Sauval, A. 1998, Space Sci. Rev., 85, 161

Hillier, D. J. 2008, Massive Stars as Cosmic Engines, Proceedings of the International Astronomical Union, IAU Symp., 250, 89

Hillier, D. J., \& Miller, D. L. 1998, ApJ, 496, 407

Hillier, D. J., Lanz, T., Heap, S. R., et al. 2003, ApJ, 588, 1039

Howarth, I. D., \& Smith, K. C. 2001, MNRAS, 327, 353

Howarth, I. D., Prinja, R. K., \& Willis, A. J. 1984, MNRAS, 208, 525

Howarth, I. D., Bolton, C. T., Crowe, R. A., et al. 1993, ApJ, 417, 338

Howarth, I. D., Siebert, K. W., Hussain, G. A. J., \& Prinja, R. K. 1997, MNRAS, 284,265

Hubeny, I., \& Lanz, T. 1995, ApJ, 439, 875

Jankov, S., Janot-Pacheco, E., \& Leister, N. V. 2000, ApJ, 540, 535

Kaltcheva, N. T., \& Hilditch, R. W. 2000, MNRAS, 312, 753

Kaufer, A., Stahl, O., Tubbesing, S., et al. 1999, Messenger, 95, 8

Krtička, J., \& Kubát, J. 2009 [arXiv: 0901. 0223]

Kudrizki, R.-P., \& Puls, J. 2000, ARA\&A, 38, 613

Lamers, H. J. G. L. M., Haser, S., de Koter, A., \& Leitherer, C. 1999, ApJ, 516, 872

Lanz, T., \& Hubeny, I. 2003, ApJS, 146, 417

Lanz, T., \& Hubeny, I. 2007, ApJS, 169, 83

Leitherer, C. 1988, ApJ, 326, 356

Lesh, J. R. 1968, ApJS, 17, 371

Lucy, L. B., \& Solomon, P. M. 1970, ApJ, 159, 879

MacConnell, D. J., \& Bidelman, W. P. 1976, AJ, 81, 225

Macfarlane, J. J., Cohen, D. H., \& Wang, P. 1994, ApJ, 437, 351

Maíz-Apellániz, J., Walborn, N. R., Galué, H. A., \& Wei, L. H. 2004, ApJS, 151, 103

Martins, F., Schaerer, D., \& Hillier, D. J. 2002, A\&A, 382, 999

Martins, F., Schaerer, D., Hillier, D. J., \& Heydari-Malayeri, M. 2004, A\&A, 420, 1087

Martins, F., Schaerer, D., Hillier, D. J., et al. 2005a, A\&A, 441, 735

Martins, F., Schaerer, D., \& Hillier, D. J. 2005b, A\&A, 436, 1049

Massey, P. 2003, ARA\&A, 41, 15
Mathys, G., Andrievsky, S. M., Barbuy, B., Cunha, K., \& Korotin, S. A. 2002, A\&A, 387, 890

Meynet, G., \& Maeder, A. 2000, A\&A, 361, 101

Mokiem, M. R., de Koter, A., Puls, J., et al. 2005, A\&A, 441, 711

Mokiem, M. R., de Koter, A., Vink, J. S., et al. 2007, A\&A, 473, 603

Moultaka, J., Ilovaisky, S. A., Prugniel, P., \& Soubiran, C. 2004, PASP, 116, 693

Niemela, V. S., \& Méndez, R. H. 1974, ApJ, 187, L23

Penny, L. R. 1996, ApJ, 463, 737

Prinja, R. K., Massa, D., \& Searle, S. C. 2005, A\&A, 430, L41

Puls, J. 2008, Massive Stars as Cosmic Engines, Proceedings of the International Astronomical Union, IAU Symp., 250, 25

Puls, J., Urbaneja, M. A., Venero, R., et al. 2005, A\&A, 435, 669

Puls, J., Markova, N., Scuderi, S., et al. 2006, A\&A, 454, 625

Oskinova, L. 2005, MNRAS, 361, 679

Oskinova, L., Feldmeier, A., \& Hamann, W.-R. 2006, MNRAS, 372, 313

Owocki, S. P., Castor, J. I., \& Rybicki, G. B. 1988, ApJ, 335, 914

Reed, B. C. 1993, PASP, 105, 1465

Reid, A. H. N., Bolton, C. T., Crowe, R. A., et al. 1993, ApJ, 417, 320

Repolust, T., Puls, J., \& Herrero, A. 2004, A\&A, 415, 349

Sana, H., Rauw, G., Nazé, Y., Gosset, E., \& Vreux, J.-M. 2006, MNRAS, 372, 661

Sana, H., Nazé, Y., O’Donnell, B., Rauw, G., \& Gosset, E. 2008, New Astron., 13,202

Schild, R. E., Hiltner, W. A., \& Sanduleak, N. 1969, ApJ, 156, 609

Searle, S. C., Prinja, R. K., Massa, D., \& Ryans, R. 2008, A\&A, 481, 777

Smith, N., \& Owocki, S. P. 2006, ApJ, 645, L45

Snow, T. P., \& Jenkins, E. B. 1977, ApJS, 33, 269

Snow, T. P., Lamers, H. J. G. L. M., Lindholm, D. M., \& Odell, A. P. 1994, ApJS, 95,163

Ud-Doula, A., \& Owocki, S. P. 2002, ApJ, 576, 413

Vacca, W. D., Garmany, C. D., \& Shull, J. M. 1996, ApJ, 460, 914

van Marle, A. J., Owocki, S. P., \& Shaviv, N. J. 2008, MNRAS, 389, 1353

Villamariz, M. R., \& Herrero, A. 2005, A\&A, 442, 263

Vink, J. S., de Koter, A., \& Lamers, H. J. G. L. M. 1999, A\&A, 350, 181

Vink, J. S., de Koter, A., \& Lamers, H. J. G. L. M. 2000, A\&A, 362, 295

Vink, J. S., de Koter, A., \& Lamers, H. J. G. L. M. 2001, A\&A, 369, 574

Walborn, N. R. 1973, AJ, 78, 1067

Walborn, N. R., \& Panek, R. J. 1984, ApJ, 286, 718

Waldron, W. L., \& Cassinelli, J. P. 2009, ApJL, 692, 76

Walker, G. A. H., Yang, S., \& Fahlman, G. G. 1979, ApJ, 233, 199

Walker, G. A. H., Kuschnig, R., Matthews, J. M., et al. 2005, ApJ, 623, L145

Woosley, S. E., \& Bloom, J. S. 2006, ARA\&A, 44, 507

Zsargó, J., Hillier, D. J., Bouret, J.-C., et al. 2008, ApJ, 685, L149 


\section{Appendix A: Model fits}

In this section, we show our best fits to the observed spectra.
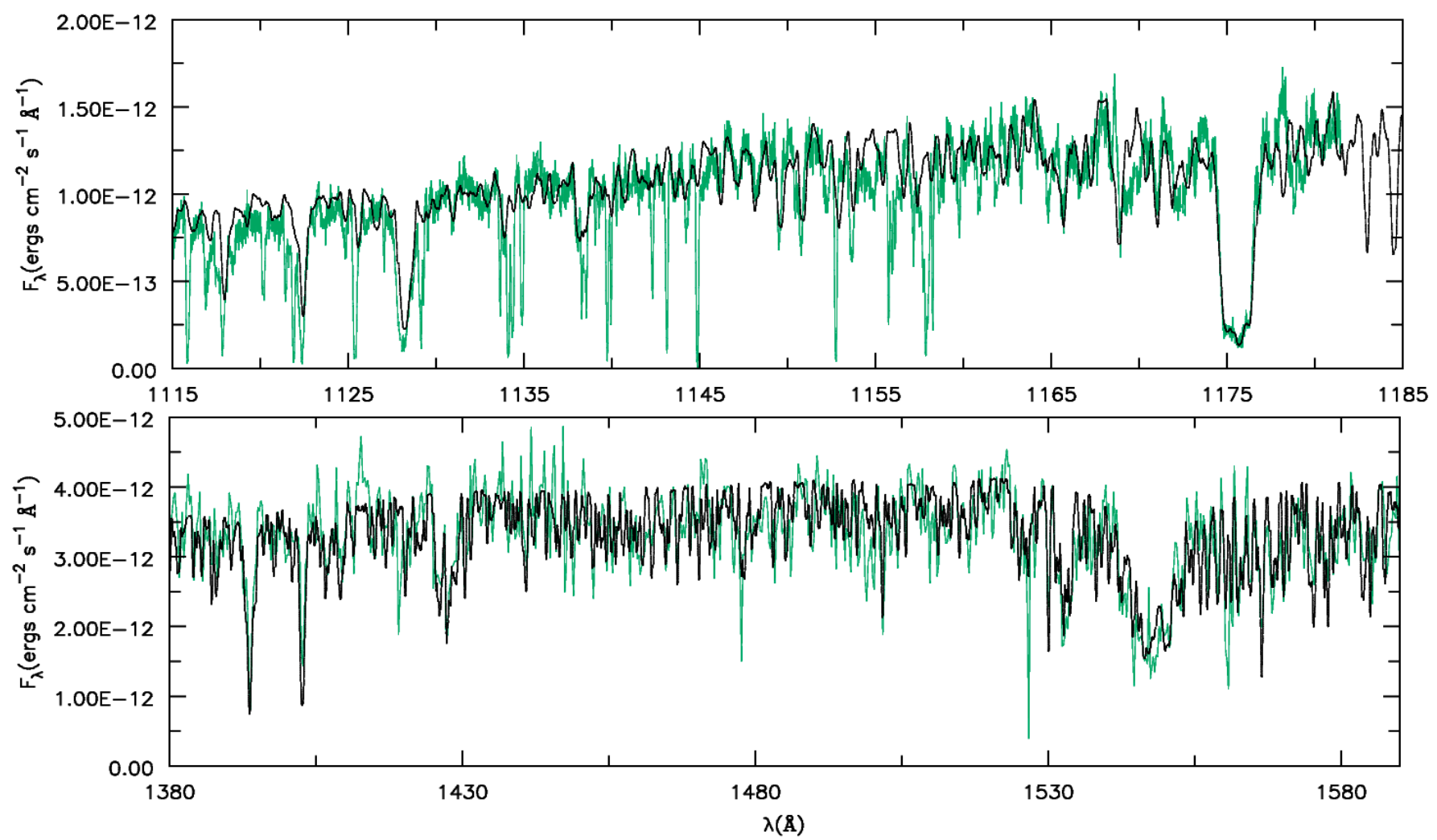

Fig. A.1. Ultraviolet spectra of HD 216898 (green/light gray line) and our final model (black line; $\log \dot{M}=-9.35$ ). 
W. L. F. Marcolino et al.: Analysis of late-type O dwarfs, Online Material p 2
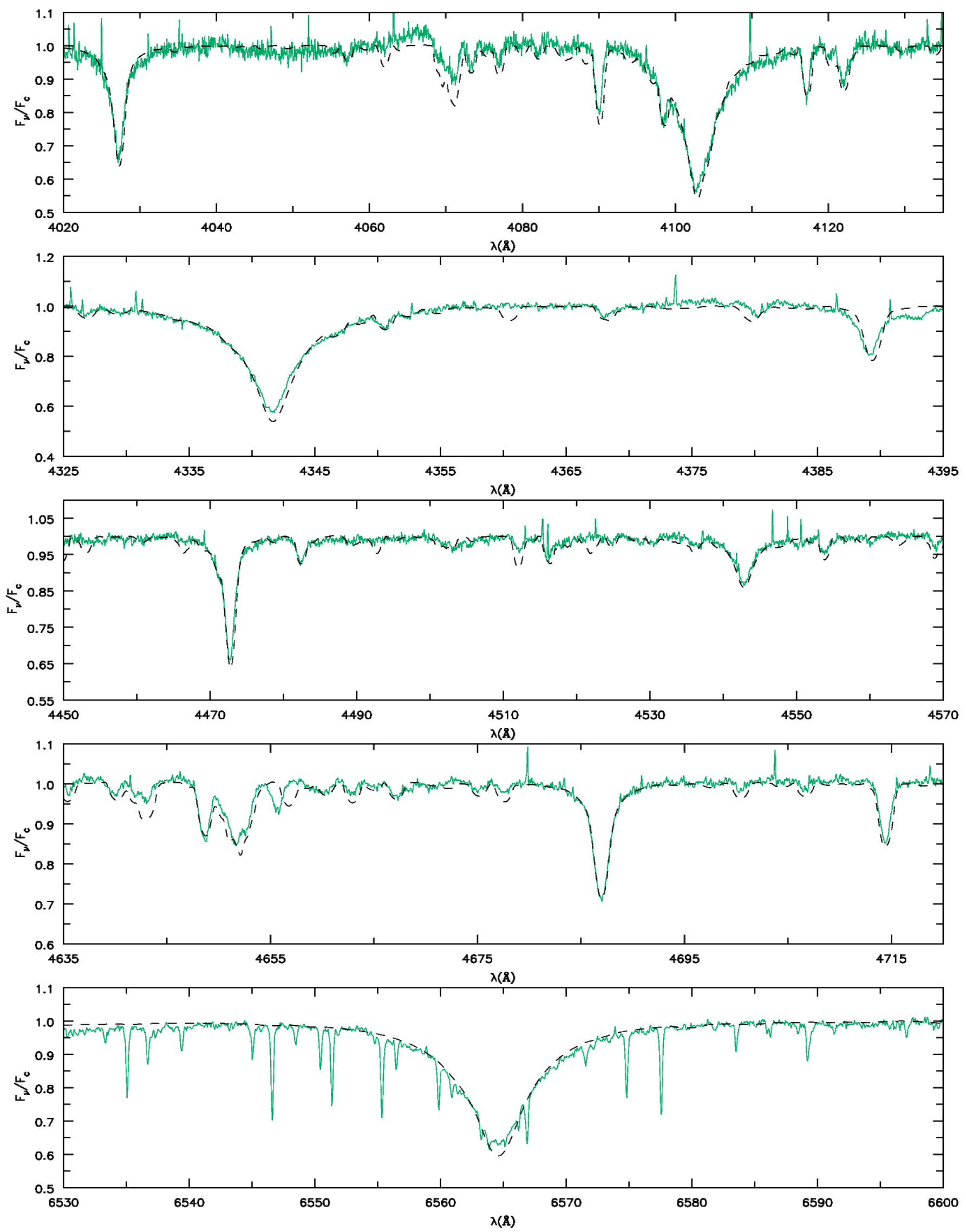

Fig. A.2. Optical spectrum of HD 216898 (green/light gray line) and our final model (black/dashed line). 
W. L. F. Marcolino et al.: Analysis of late-type O dwarfs, Online Material p 3
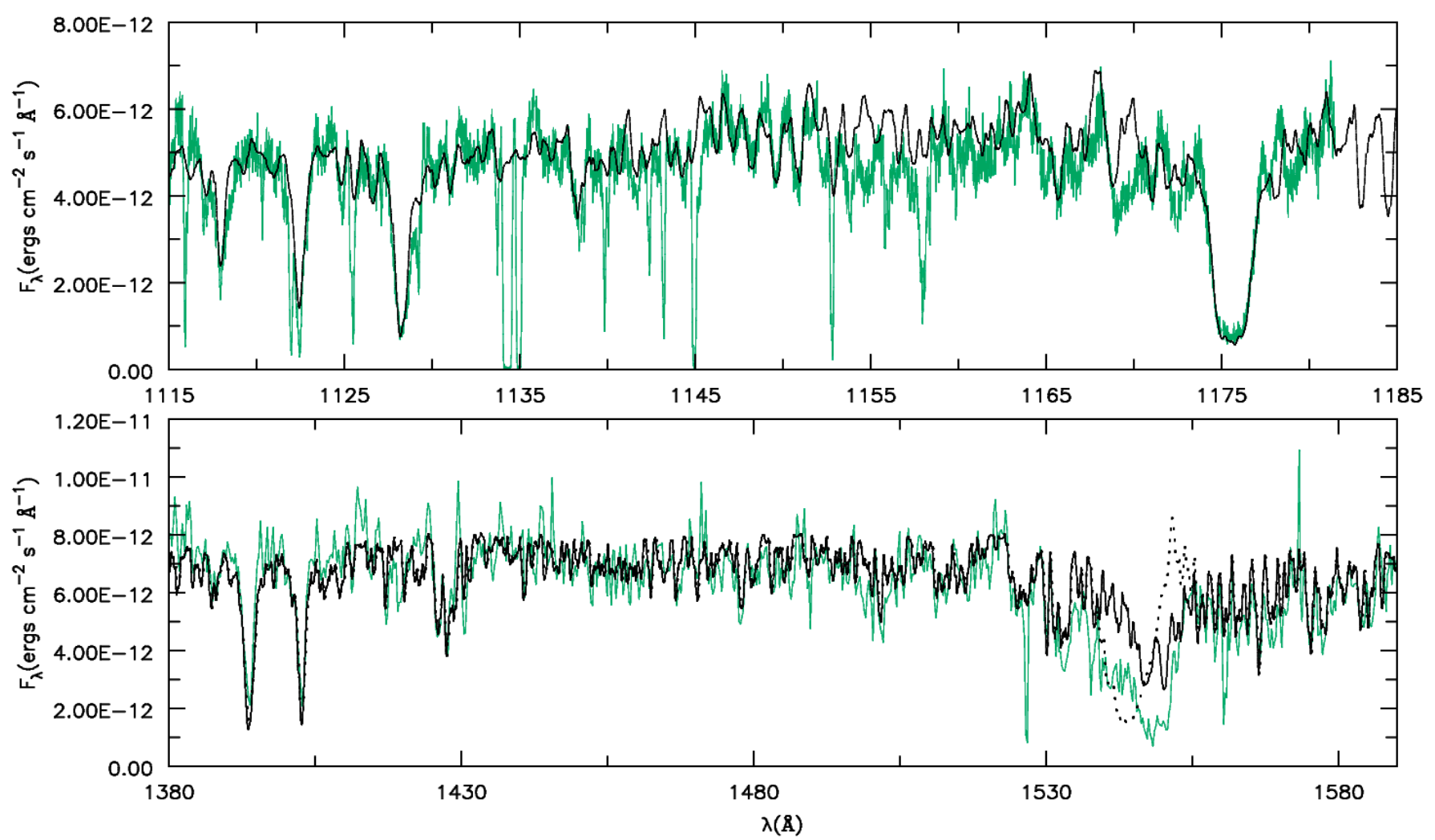

Fig. A.3. Ultraviolet spectra of HD 326329 (green/light gray line) and our final model (black $\operatorname{line} ; \log \dot{M}=-9.22$ ). A model with a higher mass-loss rate is shown as a dotted $\operatorname{line}(\log \dot{M}=-8.55$; see text for more details). 
W. L. F. Marcolino et al.: Analysis of late-type O dwarfs, Online Material p 4
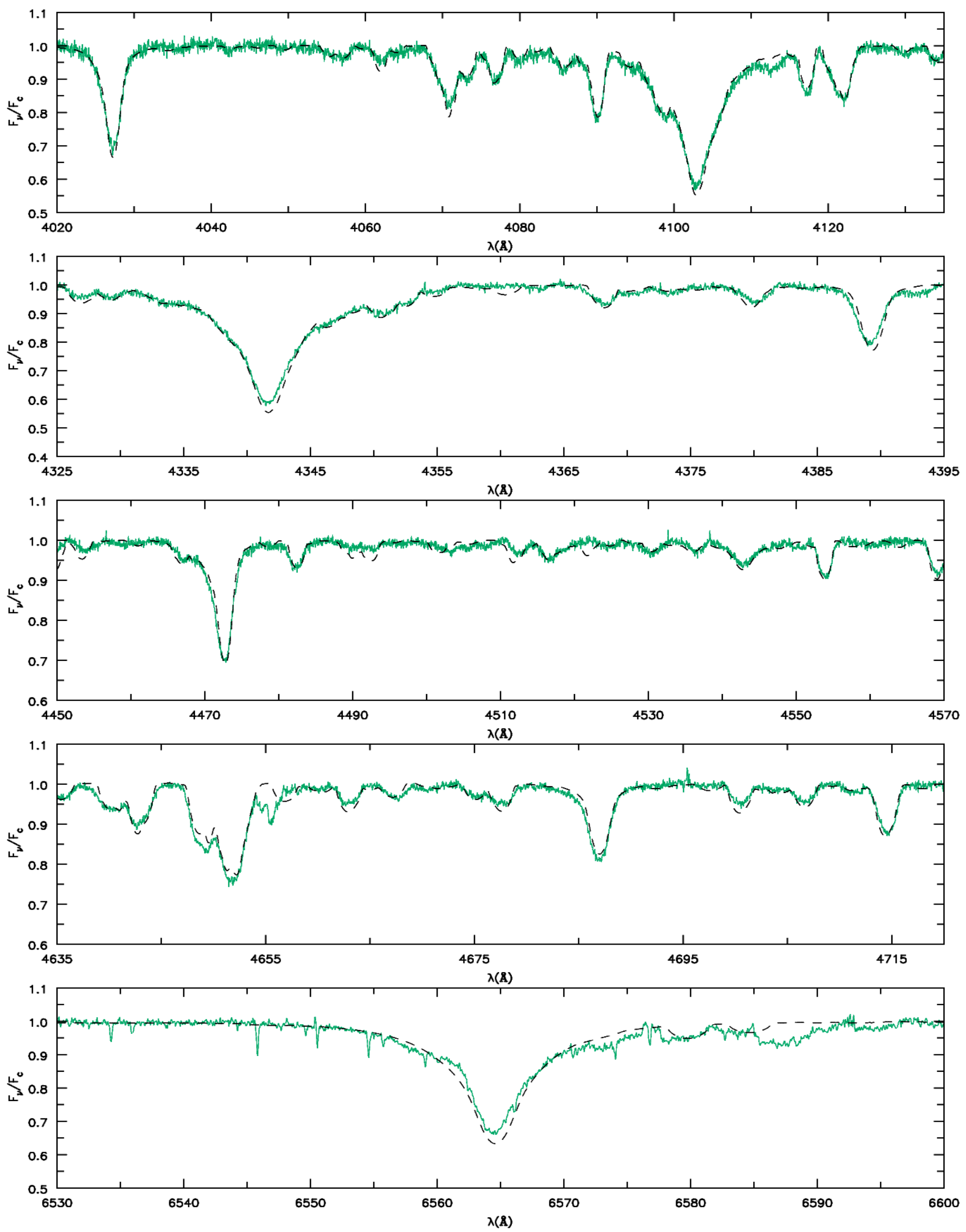

Fig. A.4. Optical spectrum of HD 326329 (green/light gray line) and our final model (black/dashed line). 
W. L. F. Marcolino et al.: Analysis of late-type O dwarfs, Online Material p 5

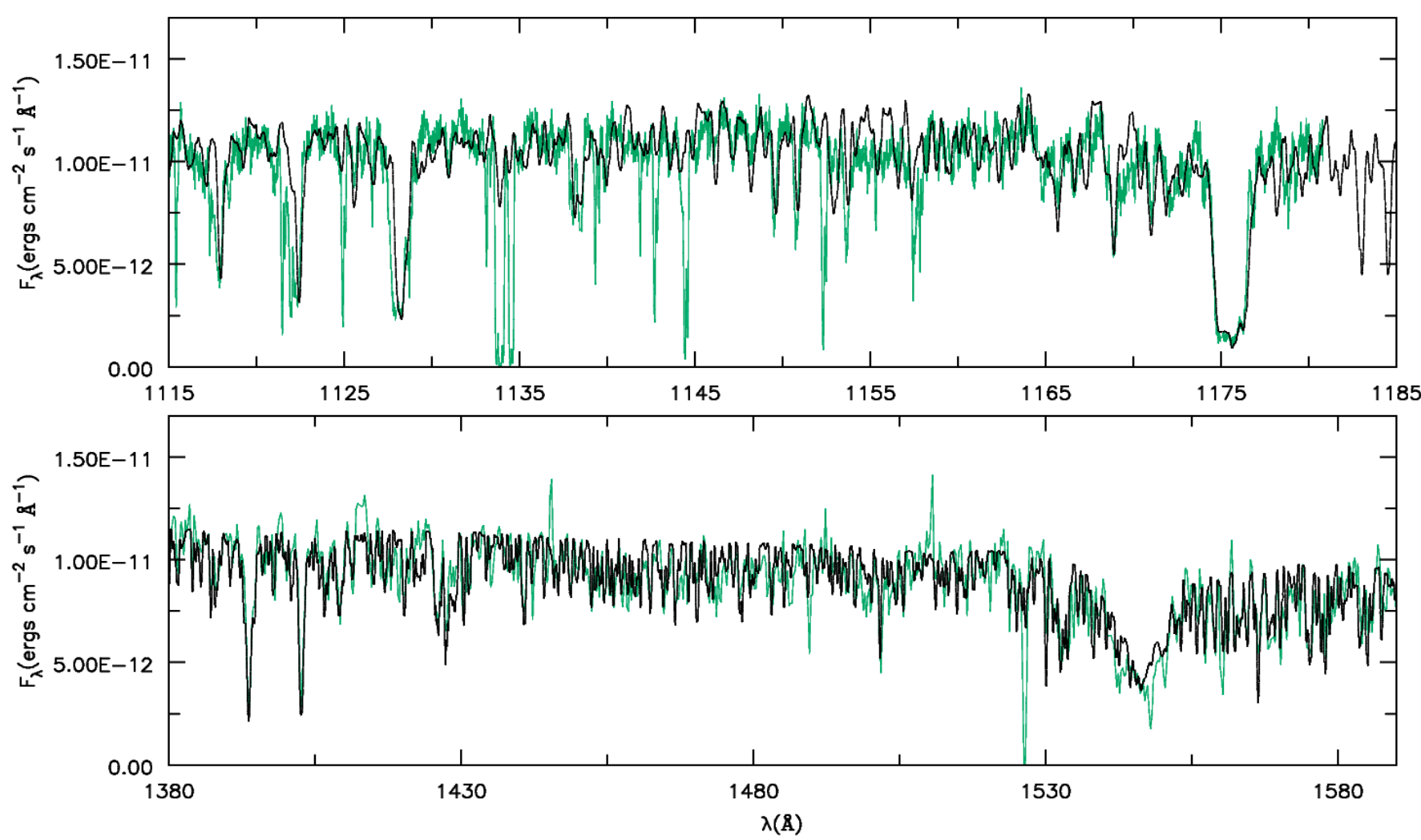

Fig. A.5. Ultraviolet spectra of HD 66788 (green/light gray line) and our final model (black line; $\log \dot{M}=-8.92$ ). 
W. L. F. Marcolino et al.: Analysis of late-type O dwarfs, Online Material p 6
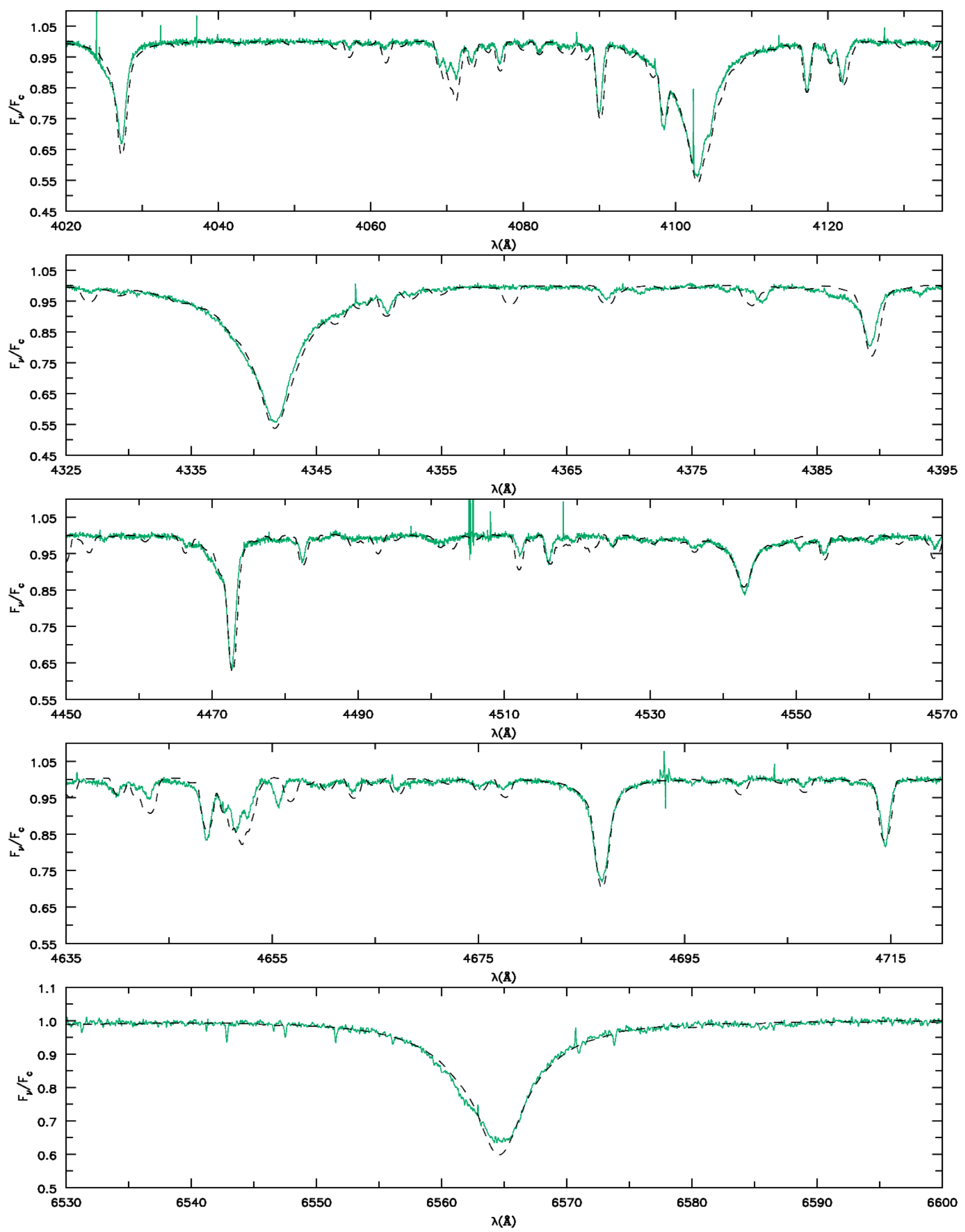

Fig. A.6. Optical spectrum of HD 66788 (green/light gray line) and our final model (black/dashed line). 
W. L. F. Marcolino et al.: Analysis of late-type O dwarfs, Online Material $p 7$
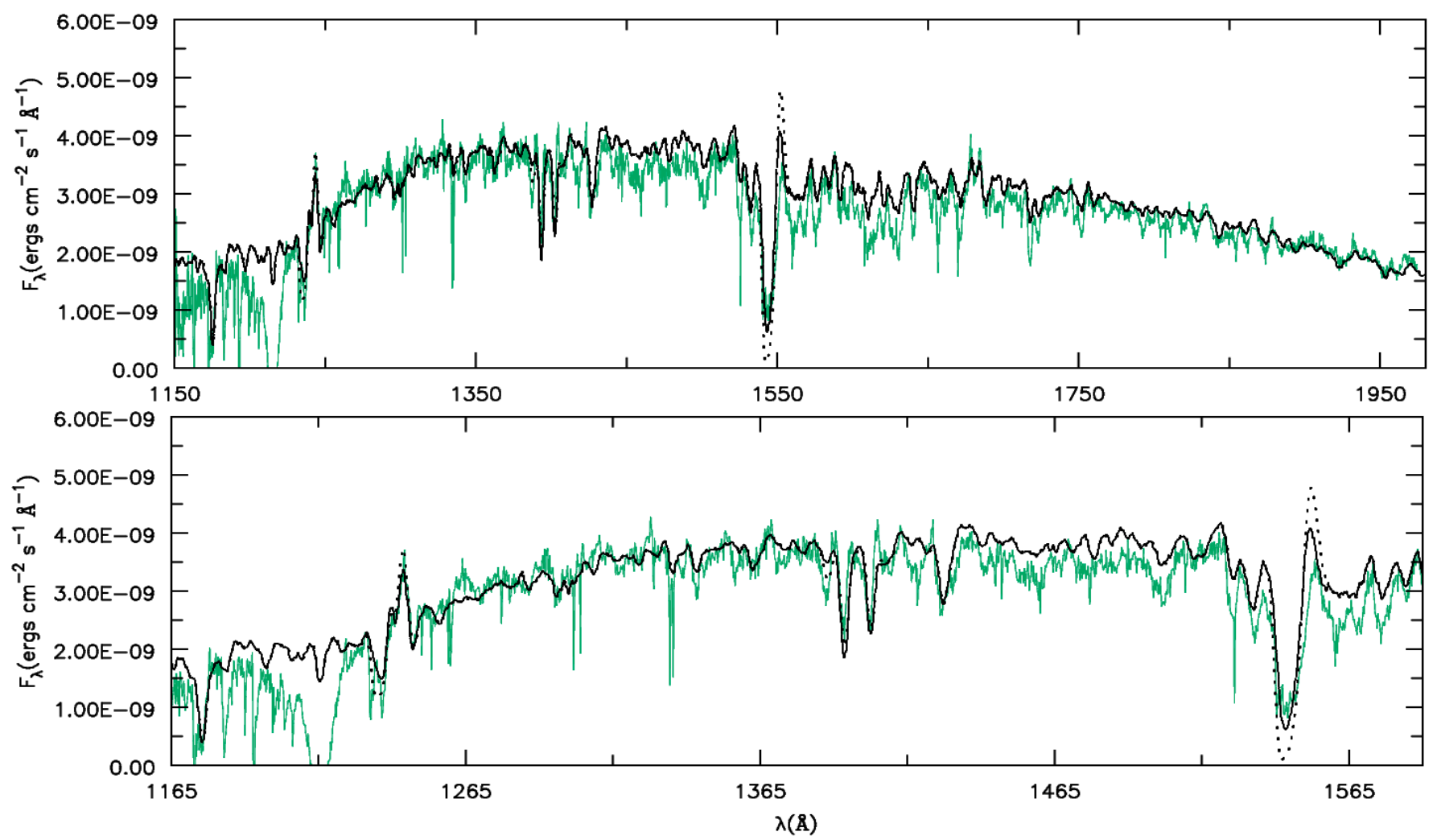

Fig. A.7. Ultraviolet spectrum of $\zeta$ Oph (green/light gray line) and our final model (black line; $\log \dot{M}=-8.80$ ). A model with a higher mass-loss rate is shown as a dotted line $(\log \dot{M}=-8.30$; see text for more details). 
W. L. F. Marcolino et al.: Analysis of late-type O dwarfs, Online Material $p 8$
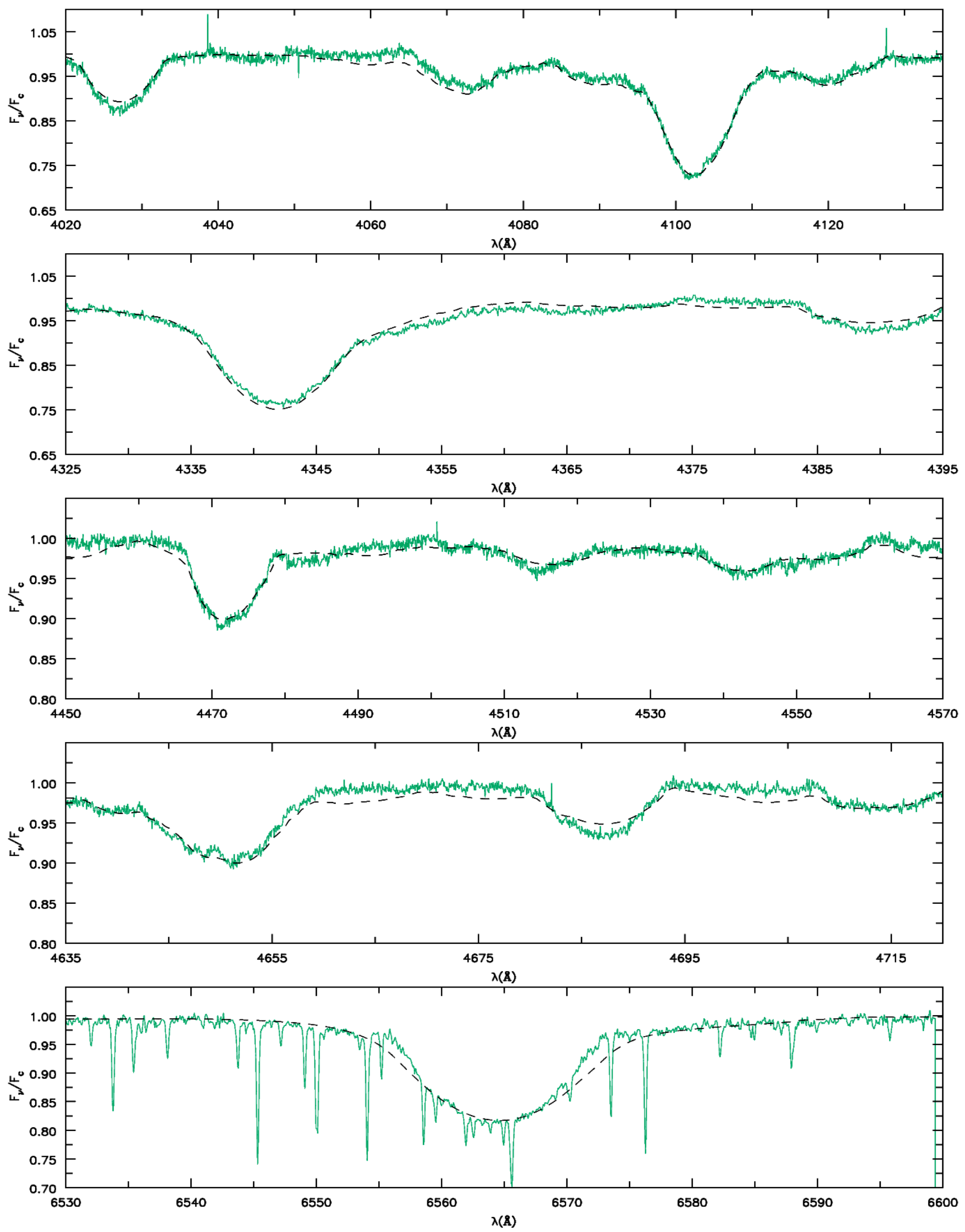

Fig. A.8. Optical spectrum of $\zeta$ Oph (green/light gray line) and our final model (black/dashed line). 
W. L. F. Marcolino et al.: Analysis of late-type O dwarfs, Online Material p 9
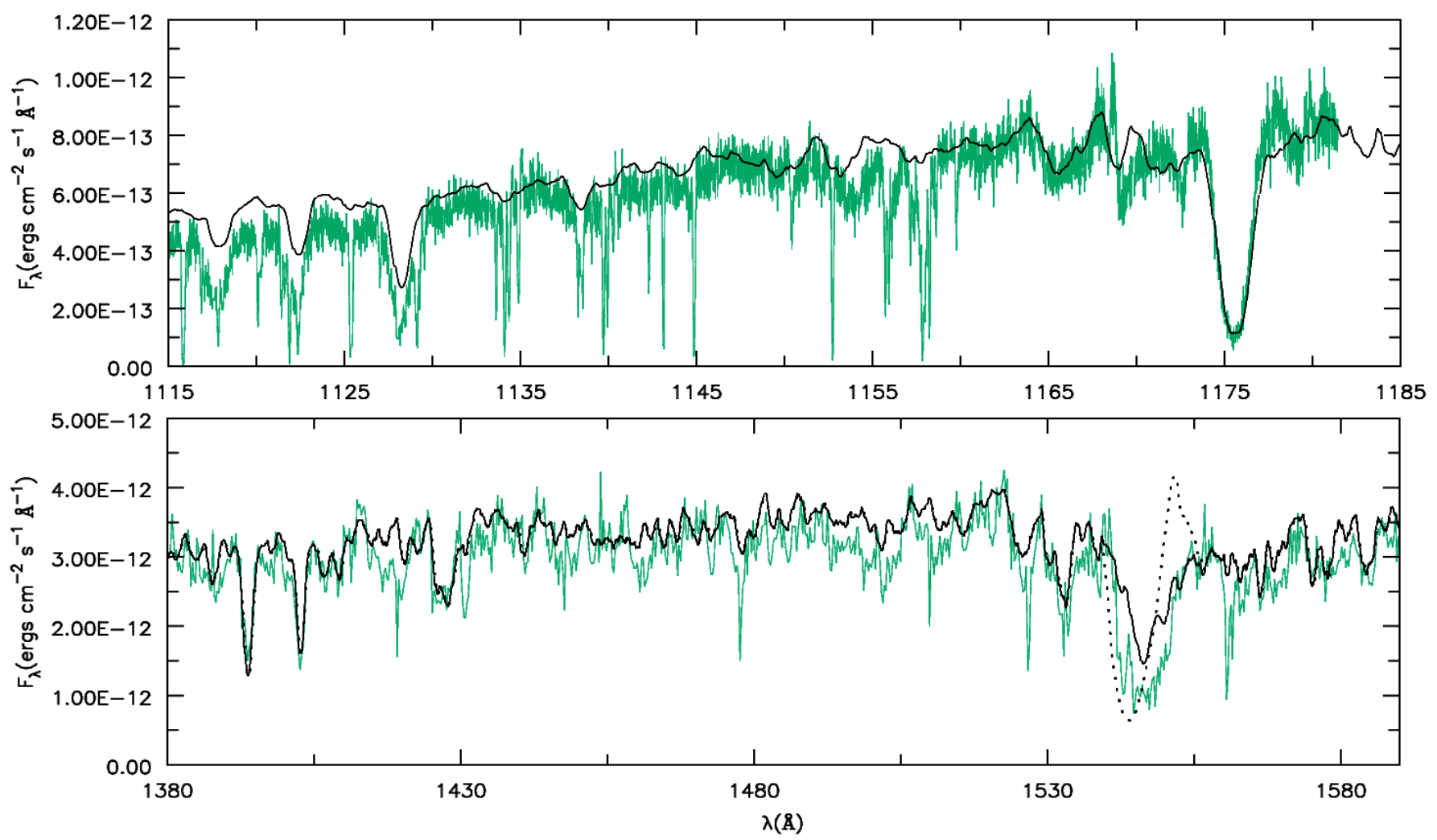

Fig. A.9. Ultraviolet spectra of HD 216532 (green/light gray line) and our final model (black $\operatorname{line} ; \log \dot{M}=-9.22$ ). A model with a higher mass-loss rate is shown as a dotted $\operatorname{line}(\log \dot{M}=-8.78$; see text for more details). 
W. L. F. Marcolino et al.: Analysis of late-type O dwarfs, Online Material p 10
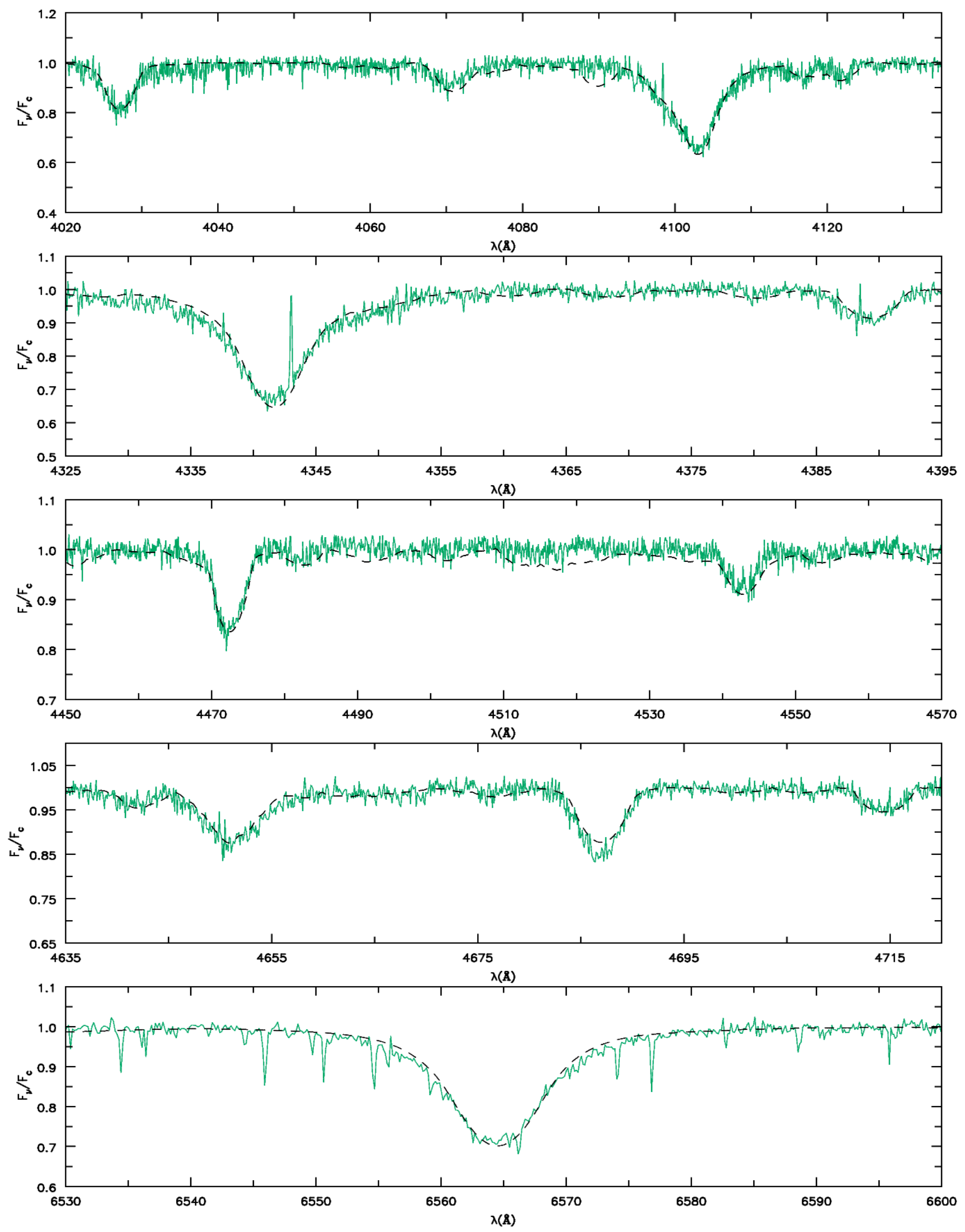

Fig. A.10. Optical spectrum of HD 216532 (green/light gray line) and our final model (black/dashed line). 


\section{Appendix B: $\dot{M}$ upper limits}

In this section, we present the model fits used to establish upper limits on the mass-loss rate from different UV lines.

- HD 216898 (Fig. B.1): we show the far-UV and UV observed spectra together with our final model; the models that determine the upper limits in $\dot{M}$ from each line $\left(\dot{M}_{\text {upper }}\right.$; not necessarily the same for each line); and the model computed with the theoretical mass-loss rate $\left(\dot{M}_{\text {Vink }}\right)$. As readily apparent from Fig. B.1: (i) the final model presents the best fit to the observed spectra; (ii) the model with $\dot{M}_{\text {Vink }}$ shows the largest discrepancy; and (iii) the models with the upper limits for the mass-loss rate do not provide satisfactory fits, and hence provide conservative upper limits. These conclusions are also valid for the objects discussed below. As we increased $\dot{M}$, the $\mathrm{N}$ v $\lambda \lambda 1239,1243$ line quickly turned into a P-Cygni profile and remained essentially unchanged, even as we increased $\dot{M}$ towards $\dot{M}_{\text {Vink }}$.

- HD 326329 (Fig. B.2): as for HD 216898. For this star the N IV $\lambda 1718$ transition is insensitive to $\dot{M}$, and the derived upper limit for $\dot{M}$ is close to $\dot{M}_{\text {Vink }}$. For the other lines the upper limits are significantly lower than $\dot{M}_{\text {Vink }}$.

- HD 66788 (Fig. B.3): the same line trends found for HD 326329 and HD 216898 are observed.

- $\zeta$ Oph (Fig. B.4): for N IV $\lambda 1718$ our final model does not yield an adequate fit to the observed line, which is considerably deeper than in the model. None of the explored $\dot{M}$ values could fit this feature, but with increasing mass-loss rate the absorption still became blue-shifted (as in the other objects). We conservatively assume that the mass-loss rate upper limit from this line equals Vink's mass-loss rate. Another difficult situation was presented by $\mathrm{N} v \lambda \lambda 1239,1243$; the model using $\dot{M}_{\text {Vink }}$ (the largest mass-loss rate) has a synthetic P-Cygni emission less intense than the one computed with the upper limit model. A slightly stronger emission is, however, found at $\sim 1249 \AA$. To choose the upper limit model we focused on the absorption component of the P-Cygni profile. The theoretical line is deeper than observed, especially around $1235 \AA$. We did not consider the two deep absorption components, indicated by two vertical lines in Fig. B.4, in the analysis. These features show significant variability in $\zeta$ Oph (mainly the bluest one), and are interpreted as being due to DACs (Howarth et al. 1993; see their Fig. 2). The situation for P $\mathrm{V} \lambda \lambda 1118,1128$ and $\mathrm{Si}$ IV $\lambda \lambda 1394,1403$ is somewhat simpler. Although our final model does not show a very good match to the observed lines, it correctly predicts them in absorption. The same does not happen for the models computed using the upper limit mass-loss rate and $\dot{M}_{\text {Vink }}$. - HD 216532 (Fig. B.5): the C III $\lambda 1176$, P v $\lambda \lambda 1118,1128$, and Si IV $\lambda \lambda 1394,1403$ lines show well developed P-Cygni profiles with $\dot{M}_{\text {Vink }}$, and these are not observed. For the $\mathrm{P} V \lambda \lambda 1118,1128$ lines, the final model fit is not deep enough. Nevertheless, an upper limit for $\dot{M}$ can be derived without difficulty as these lines turn to emissions when $\dot{M}$ is increased, and this is not what is observed. 
W. L. F. Marcolino et al.: Analysis of late-type O dwarfs, Online Material p 12

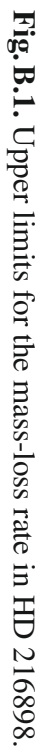

normalized flux
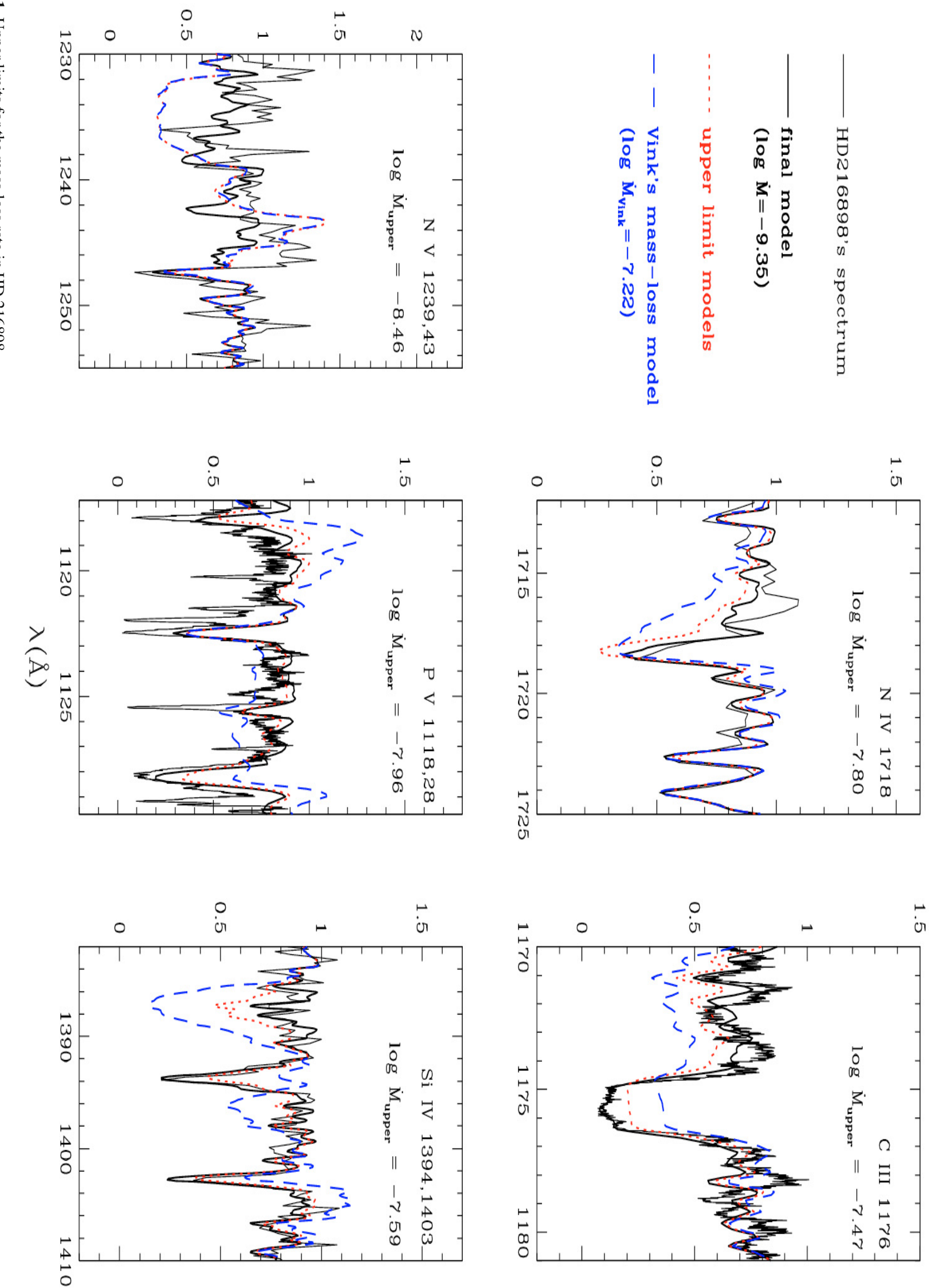
W. L. F. Marcolino et al.: Analysis of late-type O dwarfs, Online Material p 13

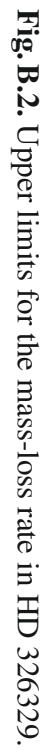

normalized flux
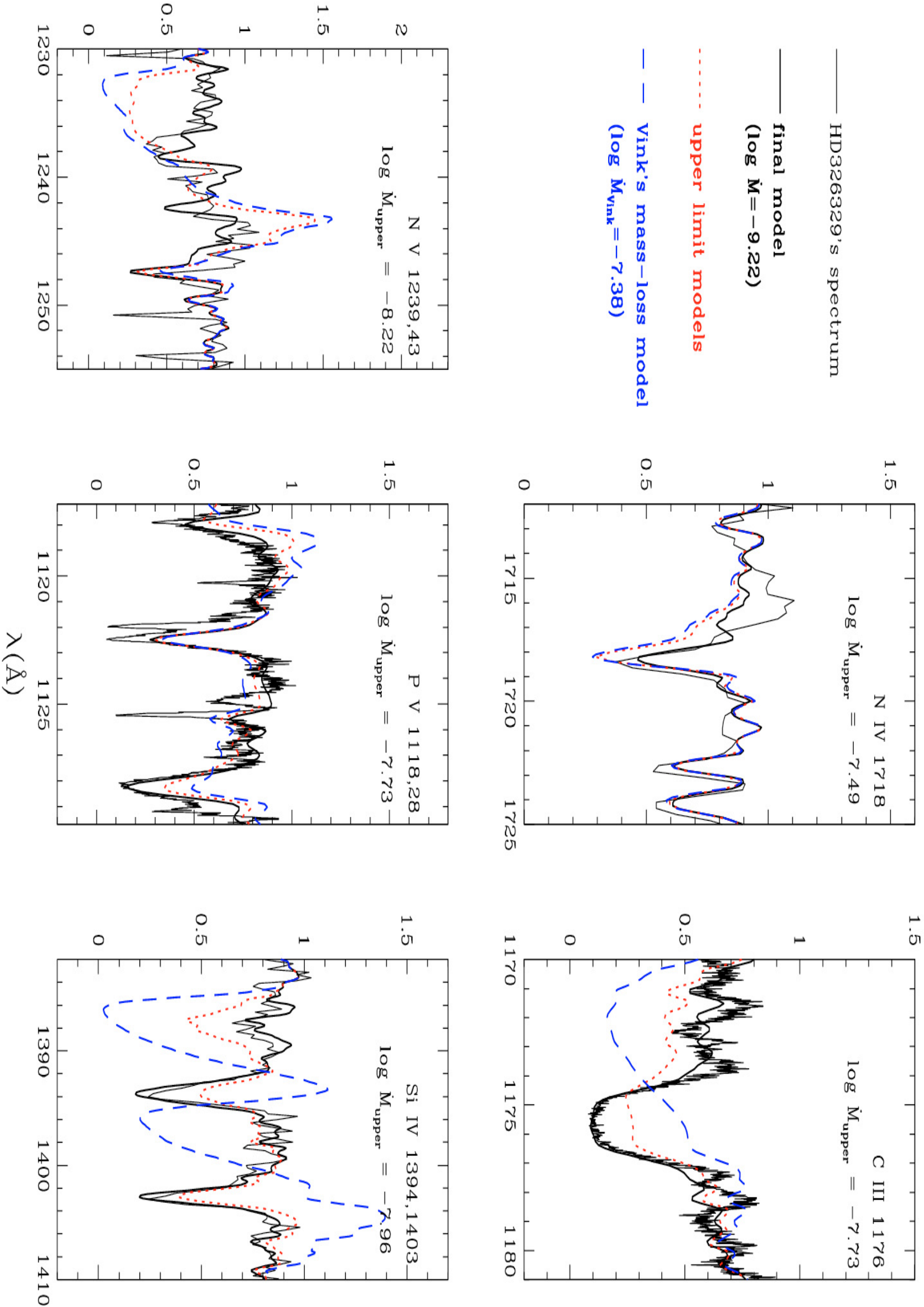
W. L. F. Marcolino et al.: Analysis of late-type O dwarfs, Online Material p 14

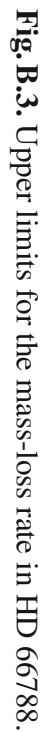

normalized flux
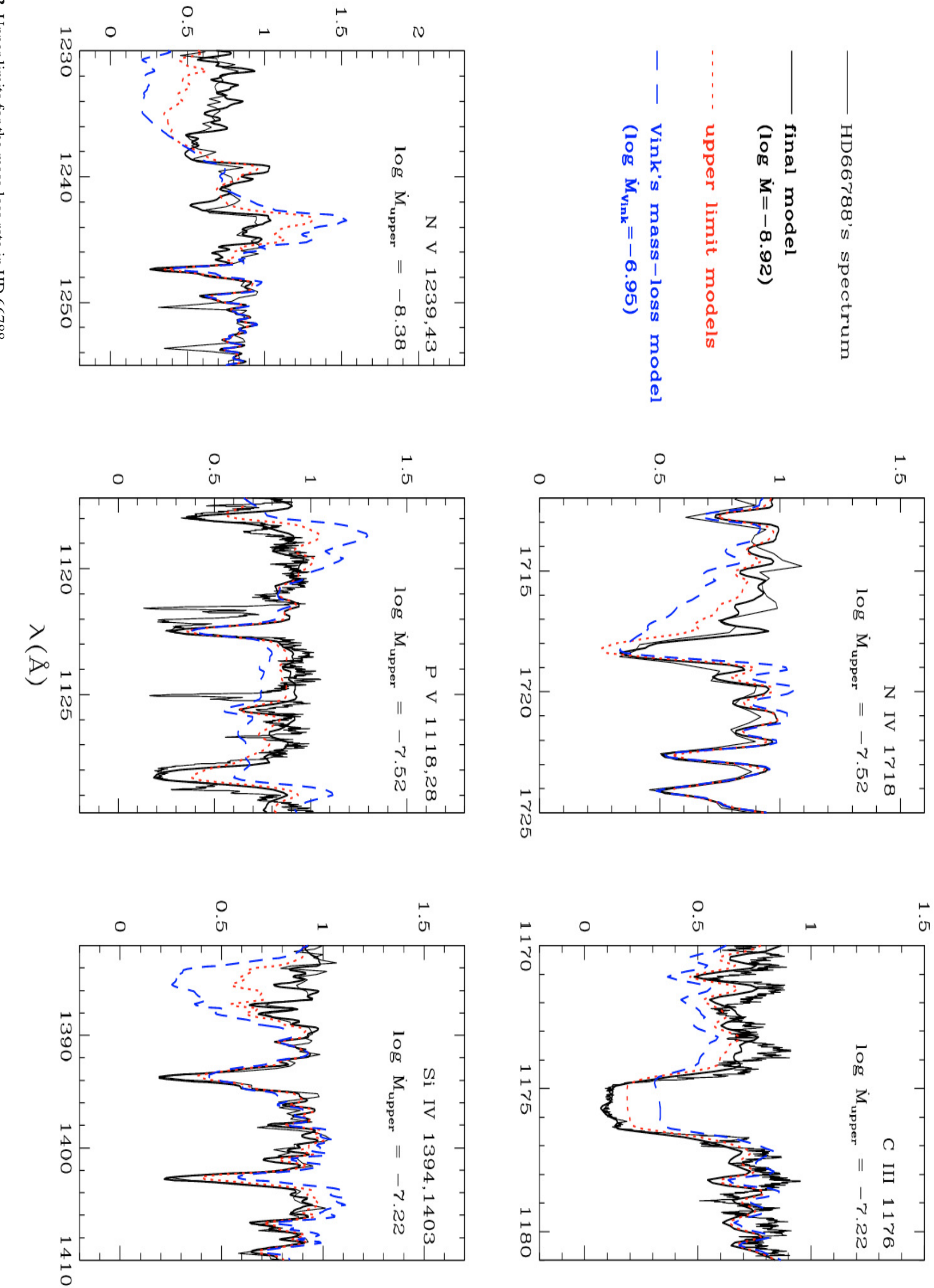
W. L. F. Marcolino et al.: Analysis of late-type O dwarfs, Online Material p 15

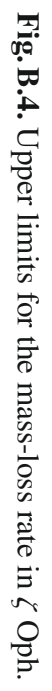
normalized flux
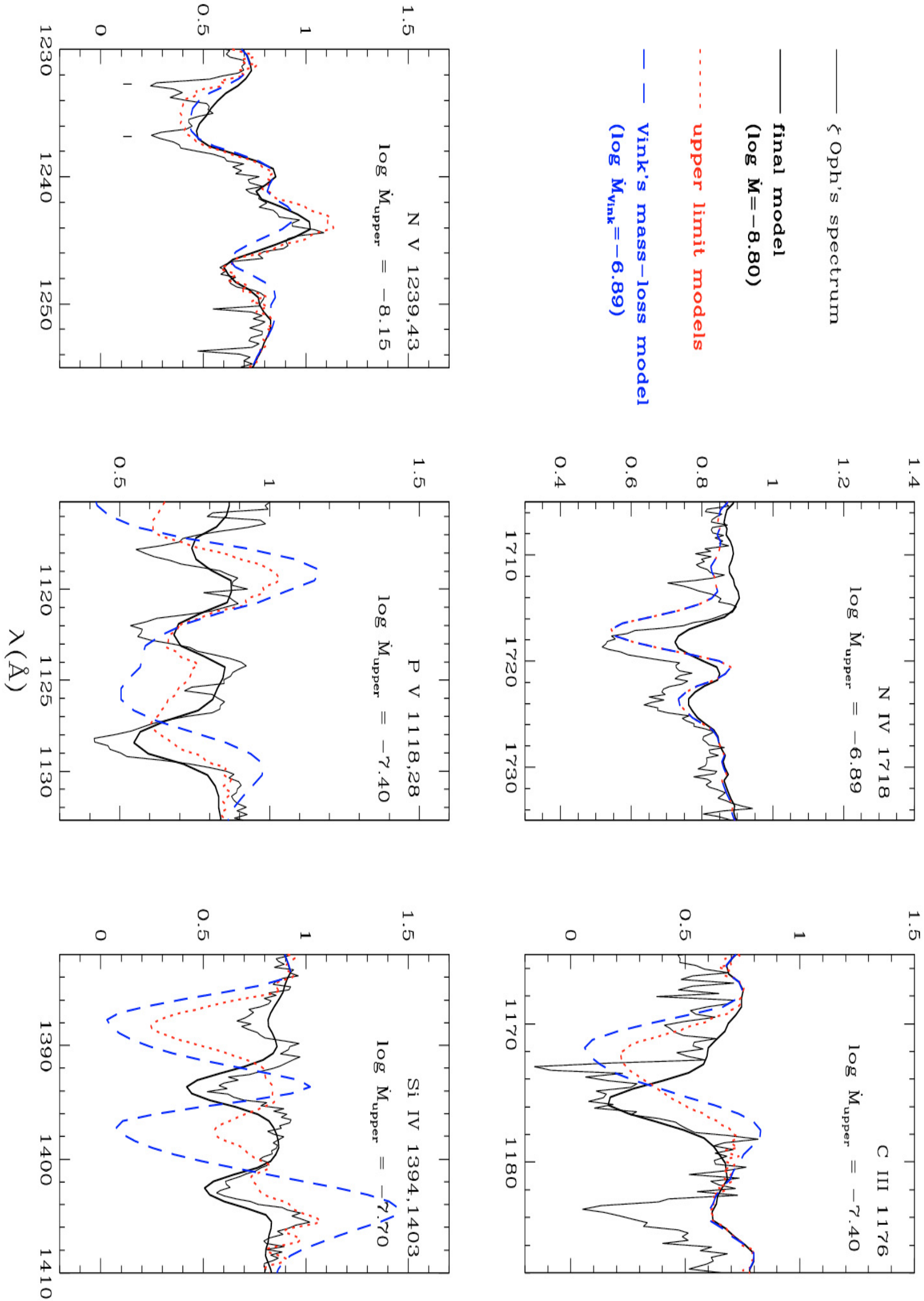
W. L. F. Marcolino et al.: Analysis of late-type O dwarfs, Online Material p 16

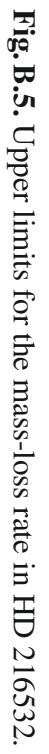
normalized flux
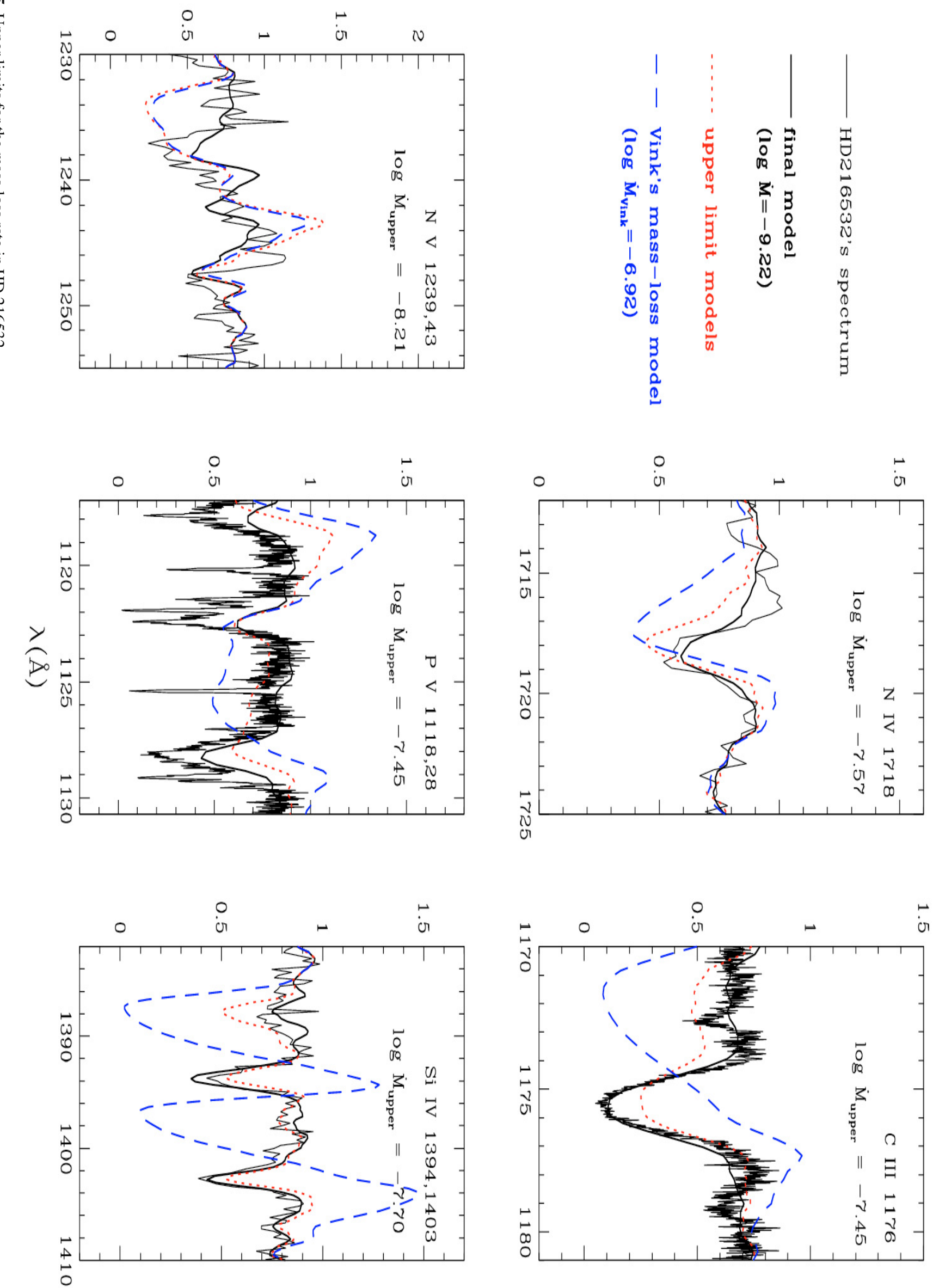